\title{
Synthesis of Heterocyclic Compounds Derived From Dimedone and their Anti-tumor and Tyrosine Kinase Inhibitions
}

\author{
Rafat M. Mohareb, ${ }^{1,}{ }^{*}$ Fatma M. Manhi ${ }^{2}$ and Amal Abdelwahab ${ }^{2}$ \\ ${ }^{1}$ Department of Chemistry, Faculty of Science, Cairo University, Giza, A. R. Egypt \\ ${ }^{2}$ National Organization for Drug Control \& Research, P.O. 29, Cairo, A. R. Egypt \\ *Corresponding author: E-mail: raafat_mohareb@yahoo.com
}

Received: 04-25-2019

\begin{abstract}
The reaction of dimedone with arylaldehydes gave the benzylidene derivatives $\mathbf{3 a}-\mathbf{c}$, the latter underwent a series of heterocyclization reactions to give fused thiophene, pyrazole isoxazole and pyridazine derivatives. The synthesized compounds were evaluated against different kinds of cancer cell lines together tyrosine kinases and Pim-1 kinase inhibitions. All the synthesized compounds were assessed for the inhibitory activities against A549 (non-small cell lung cancer), H460 (human lung cancer), HT-29 (human colon cancer) and MKN-45 (human gastric cancer) cancer cell lines together with foretinib as the positive control by a MTT assay. The promising compounds were 3c, 5b, 5e, 5f, 7c, 7f, 9c, 11b, 12c, 12d, 13b, 13d, 14b, 16c and 16d among the tested compounds. On the other hand, compounds 5b, 5e, 5f, 7c, 11b, 12c, 12d, 13d, 14b, 16c and 16d were the most effective inhibitors against tyrosine kinases and compounds 5b, 11b, 12d, 13d, $14 \mathrm{~b}$ and $\mathbf{1 6 c}$ were the most potent against Pim-1 kinase.
\end{abstract}

Keywords: Dimedone; thiophene; pyrazole; isoxazole; antitumor; tyrosine kinase

\section{Introduction}

As typical reactive 1,3-dicarbonyl compounds, cyclohexane-1,3-dione and its analog 5,5-dimethyl cyclohexane-1,3-dione (dimedone) have been widely used in versatile synthetic reactions. ${ }^{1,2}$ Dimedone is not only a typical reagent for Knoevenagel condensation, but also adds easily to electron-deficient alkenes via Michael addition. On the other hand, its one or two carbonyl groups could take part in substitution and cyclization reactions through the tautomeric enolate form. Thus, the cascade reactions of addition, elimination and substitution could be achieved in many reactions involving dimedone. The reactions of cyclohexane-1,3-dione or dimedone with aldehydes have been extensively studied in the past years, from which several types of compounds have been produced according to the reaction conditions. ${ }^{3,4}$ The normal Knoevenagel condensations of cyclohexane-1,3-done or dimedone with aldehydes have been conducted with numerous methods including promotion via amines, ${ }^{5}$ Lewis acids, ${ }^{6}$ surfactants, ${ }^{7,8}$ zeolites, ${ }^{9}$ ionic liquids. ${ }^{10}$ The use of environmentally benign methods, such as aqueous medi- $u^{11}$ or in the absence of solvents ${ }^{12}$ and the usage of ultrasound or microwave heating ${ }^{13}$ have also been developed in recent years. The reactions usually proceed further through Michael addition reaction of the second molecule of dimedone to yield tetraketones as main products. ${ }^{14}$ On the other hand, tetraketones could be easily converted to 9-substituted 1,8-dioxoxanthenes by dehydration step. ${ }^{15}$ According to our previous work a large number of heterocyclic compounds with anti-proliferative and anti-inflammatory activities were recently synthesized by our research group. ${ }^{16,17}$ Recently, our research group was involved in the synthesis and determination of the anti-proliferative properties of a large number of heterocyclic compounds. ${ }^{18,19}$ In addition, according to our continued interest in the design of new multicomponent reactions and the application in the synthesis of heterocyclic compounds we found some unprecedented reaction patterns in the reaction of dimedone (1) with aromatic aldehydes $2 \mathbf{a}-\mathbf{c}$, to produce the benzylidene derivatives $\mathbf{3 a}-\mathbf{c}$ and with aromatic diazonium salts $10 \mathbf{a}, \mathbf{b}$ to produce products which underwent heterocyclization reactions to give compounds with potential antitumor activities. 


\section{Experimental}

Dry solvents were used throughout this work. All melting points of the synthesized compounds were recorded on Büchi melting point apparatus D-545. The IR spectra ( $\mathrm{KBr}$ discs) were recorded on Bruker Vector 22 instrument. ${ }^{13} \mathrm{C}$ NMR and ${ }^{1} \mathrm{H}$ NMR spectra were measured on Bruker DPX200 instrument in DMSO- $d_{6}$ with TMS as the internal standard. Mass spectra were measured using EIMS (Shimadzu) and ESI-esquire 3000 Bruker Daltonics instrument. Elemental analyses were measured using the Micro-analytical Data center at Cairo University. All reactions were monitored by TLC on 2 $\times 5 \mathrm{~cm}$ pre-coated silica gel $60 \mathrm{~F} 254$ plates of thickness of $0.25 \mathrm{~mm}$ (Merck) for determining when the reactions were complete.

\section{1. General Procedure for the Synthesis of the Benzylidene Derivatives 3a-c}

To a solution of dimedone $(1.40 \mathrm{~g}, 0.01 \mathrm{~mol})$ in absolute ethanol $(40 \mathrm{~mL})$ containing piperidine $(0.50 \mathrm{~mL})$ any of the aldehydes: benzaldehyde (1.05 g, $0.01 \mathrm{~mol})$, 4-methoxybenzaldehyde $(1.36 \mathrm{~g}, 0.01 \mathrm{~mol})$ or 4-chlorobenzaldehyde $(1.40 \mathrm{~g}, 0.01 \mathrm{~mol})$, were added. Subsequently, the mixture was heated using the reflux conditions for $1 \mathrm{~h}$, then poured onto ice/water containing a few drops of hydrochloric acid, the solid was collected by filtration, dried and crystallized from ethanol to get the $\mathbf{3 a}-\mathbf{c}$.

\section{1. 1. 2-Benzylidene-5,5-dimethylcyclohexane- 1,3-dione (3a)}

Orange crystals from ethanol; m.p. $190-192^{\circ} \mathrm{C}$; yield 78\%. IR (KBr) cm ${ }^{-1}: 3054,2986,1689,1687,1632 .{ }^{1} \mathrm{H}$ NMR (300 MHz, DMSO- $\left.d_{6}\right) \delta 7.42-7.23\left(\mathrm{~m}, 5 \mathrm{H}, \mathrm{C}_{6} \mathrm{H}_{5}\right)$, $6.02(\mathrm{~s}, 1 \mathrm{H}, \mathrm{CH}), 2.31,2.28\left(2 \mathrm{~s}, 4 \mathrm{H}, 2 \mathrm{CH}_{2}\right), 1.09,1.06(2 \mathrm{~s}$, $\left.6 \mathrm{H}, 2 \mathrm{CH}_{3}\right) ;{ }^{13} \mathrm{C}$ NMR (DMSO- $\left.d_{6}, 75 \mathrm{MHz}\right) \delta 166.2,164.8$ (C-1, C-3), 127.3, 126.6, 124.3, $121.2\left(\mathrm{C}_{6} \mathrm{H}_{5}\right), 108.6,103.2$ $(\mathrm{CH}=\mathrm{C}), 50.6(\mathrm{C}-4), 36.2(\mathrm{C}-5), 24.4\left(2 \mathrm{CH}_{3}\right)$; EIMS: $\mathrm{m} / z$ $228[\mathrm{M}]^{+}(28 \%)$; Anal. Calcd for $\mathrm{C}_{15} \mathrm{H}_{16} \mathrm{O}_{2}$ (228.29): C, 78.92; H, 7.06\%. Found: C, 78.24; H, 6.83\%.

\section{1. 2. 2-(4-Methoxybenzylidene)-5,5- dimethylcyclohexane-1,3-dione (3b)}

Pale yellow crystals from ethanol; m.p. $140-142{ }^{\circ} \mathrm{C}$; yield $78 \%$. IR $(\mathrm{KBr}) \mathrm{cm}^{-1}: 3055,2984,1689,1688,1630 .{ }^{1} \mathrm{H}$ NMR $\left(300 \mathrm{MHz}\right.$, DMSO- $\left.d_{6}\right) \delta 7.47-7.26\left(\mathrm{~m}, 4 \mathrm{H}, \mathrm{C}_{6} \mathrm{H}_{4}\right)$, $6.06(\mathrm{~s}, 1 \mathrm{H}, \mathrm{CH}), 3.68\left(\mathrm{~s}, 3 \mathrm{H}, \mathrm{OCH}_{3}\right), 2.36,2.24(2 \mathrm{~s}, 4 \mathrm{H}$, $\left.2 \mathrm{CH}_{2}\right), 1.07,1.06\left(2 \mathrm{~s}, 6 \mathrm{H}, 2 \mathrm{CH}_{3}\right) ;{ }^{13} \mathrm{C}$ NMR (DMSO- $d_{6}, 75$ $\mathrm{MHz}) \delta 166.8,164.5$ (C-1, C-3), 127.0, 126.9, 123.7, 121.6 $\left(\mathrm{C}_{6} \mathrm{H}_{4}\right), 108.3,103.6(\mathrm{CH}=\mathrm{C}), 50.3(\mathrm{C}-4), 50.1\left(\mathrm{OCH}_{3}\right)$, 36.7 (C-5), $24.2\left(2 \mathrm{CH}_{3}\right)$; EIMS: $m / z 258[\mathrm{M}]^{+}(32 \%)$; Anal. Calcd for $\mathrm{C}_{16} \mathrm{H}_{18} \mathrm{O}_{3}$ (258.31): C, 74.39; H, 7.02\%. Found: C, $74.48 ; \mathrm{H}, 6.95 \%$.

\section{1. 3. 2-(4-Chlorobenzylidene)-5,5- dimethylcyclohexane-1,3-dione (3c)}

Pale yellow crystals from ethanol; m.p. $125-127^{\circ} \mathrm{C}$; yield $80 \%$. IR $(\mathrm{KBr}) \mathrm{cm}^{-1}: 3055,2984,1690,1688,1630 .{ }^{1} \mathrm{H}$ NMR $\left(300 \mathrm{MHz}, \mathrm{DMSO}-d_{6}\right) \delta$ 7.49-7.22 (m, 4H, $\left.\mathrm{C}_{6} \mathrm{H}_{4}\right)$, $6.07(\mathrm{~s}, 1 \mathrm{H}, \mathrm{CH}), 2.38,2.21\left(2 \mathrm{~s}, 4 \mathrm{H}, 2 \mathrm{CH}_{2}\right), 1.08,1.05(2 \mathrm{~s}$, $6 \mathrm{H}, 2 \mathrm{CH}_{3}$ ); ${ }^{13} \mathrm{C}$ NMR (DMSO- $\left.d_{6}, 75 \mathrm{MHz}\right) \delta 166.5,164.2$ (C-1, C-3), 128.3, 126.5, 123.2, $121.3\left(\mathrm{C}_{6} \mathrm{H}_{4}\right), 108.8,103.3$ $(\mathrm{CH}=\mathrm{C}), 50.6(\mathrm{C}-4), 36.8(\mathrm{C}-5), 24.5\left(2 \mathrm{CH}_{3}\right)$; EIMS: $\mathrm{m} / z$ $262[\mathrm{M}]^{+}(28 \%)$; Anal. Calcd for $\mathrm{C}_{15} \mathrm{H}_{15} \mathrm{ClO}_{2}$ (262.73): C, 68.57; H, 5.75\%. Found: C, 68.39; H, 6.02\%.

\section{2. General Procedure for the Synthesis of the 6,7-Dihydrobenzo[b] thiophene Derivatives 5a-f}

To a solution of any of $3 \mathbf{a}(2.28 \mathrm{~g}, 0.01 \mathrm{~mol}), 3 \mathbf{b}(2.58$ g, $0.01 \mathrm{~mol})$ or $3 \mathrm{c}(2.62 \mathrm{~g}, 0.01 \mathrm{~mol})$ in 1,4-dioxan $(40 \mathrm{~mL})$ containing triethylamine $(0.50 \mathrm{~mL})$ each of elemental sulfur $(0.32 \mathrm{~g}, 0.01 \mathrm{~mol})$ and either of malononitrile $(0.66 \mathrm{~g}$, $0.01 \mathrm{~mol})$ or ethyl cyanoacetate $(1.07 \mathrm{~g}, 0.01 \mathrm{~mol})$ were added. The reaction mixture was heated under reflux for 1 $\mathrm{h}$ then left to cool. The formed solid product was collected by filtration, dried and crystallized from ethanol to give 5a-f, respectively.

\section{2. 1. 2-Amino-4-benzylidene-7,7-dimethyl-5- oxo- $4,5,6,7$-tetrahydrobenzo $[b]$ thiophene- 3-carbonitrile (5a)}

Yellow crystals from ethanol; m.p. $199-202^{\circ} \mathrm{C}$; yield $80 \%$. IR ( $\mathrm{KBr}) \mathrm{cm}^{-1}: 3487-3348\left(\mathrm{NH}_{2}\right), 3055,2985,1688$, 1632. ${ }^{1} \mathrm{H}$ NMR (300 MHz, DMSO- $\left.d_{6}\right) \delta 7.40-7.25$ (m, $\left.5 \mathrm{H}, \mathrm{C}_{6} \mathrm{H}_{5}\right), 6.06(\mathrm{~s}, 1 \mathrm{H}, \mathrm{CH}), 4.30\left(\mathrm{~s}, 2 \mathrm{H}, \mathrm{D}_{2} \mathrm{O}\right.$ exchangeable, $\left.\mathrm{NH}_{2}\right), 2.32\left(\mathrm{~s}, 2 \mathrm{H}, \mathrm{CH}_{2}\right), 1.08,1.06\left(2 \mathrm{~s}, 6 \mathrm{H}, 2 \mathrm{CH}_{3}\right)$; ${ }^{13} \mathrm{C}$ NMR (DMSO- $\left.d_{6}, 75 \mathrm{MHz}\right) \delta 166.0(\mathrm{C}-1), 138.6$, $136.0,135.2,130.7,127.8,125.4,124.3,121.0\left(\mathrm{C}_{6} \mathrm{H}_{5}\right.$, thiophene $\mathrm{C}), 116.9(\mathrm{CN}), 108.9,103.5(\mathrm{CH}=\mathrm{C}), 50.8(\mathrm{C}-4)$,

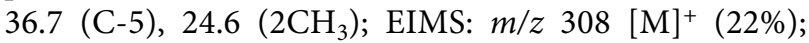
Anal. Calcd for $\mathrm{C}_{18} \mathrm{H}_{16} \mathrm{~N}_{2} \mathrm{OS}$ (308.40): C, 70.10; $\mathrm{H}, 5.23$; N, 9.08; S,10.40\%. Found: C, 70.06; H, 5.39; N, 9.29; S, $10.31 \%$.

\section{2. 2. Ethyl 2-Amino-4-benzylidene-7,7- dimethyl-5-oxo-4,5,6,7-tetrahydrobenzo[b] thiophene-3-carboxylate (5b)}

Yellow crystals from ethanol; m.p. $117-120^{\circ} \mathrm{C}$; yield 72\%. IR ( $\mathrm{KBr}) \mathrm{cm}^{-1}: 4682,3367\left(\mathrm{NH}_{2}\right), 3055,2985,1702$, 1688, 1632. ${ }^{1} \mathrm{H}$ NMR $\left(300 \mathrm{MHz}, \mathrm{DMSO}-d_{6}\right) \delta 7.43-7.27$ $\left(\mathrm{m}, 5 \mathrm{H}, \mathrm{C}_{6} \mathrm{H}_{5}\right), 6.05(\mathrm{~s}, 1 \mathrm{H}, \mathrm{CH}), 4.35\left(\mathrm{~s}, 2 \mathrm{H}, \mathrm{D}_{2} \mathrm{O}\right.$ exchangeable, $\mathrm{NH}_{2}$ ), 4.21 (q, $2 \mathrm{H}, J=7.26 \mathrm{~Hz}, \mathrm{OCH}_{2} \mathrm{CH}_{3}$ ), $2.35\left(\mathrm{~s}, 2 \mathrm{H}, \mathrm{CH}_{2}\right), 1.12\left(\mathrm{t}, 3 \mathrm{H}, J=7.26 \mathrm{~Hz}, \mathrm{OCH}_{2} \mathrm{CH}_{3}\right)$, $1.07,1.03\left(2 \mathrm{~s}, 6 \mathrm{H}, 2 \mathrm{CH}_{3}\right) ;{ }^{13} \mathrm{C}$ NMR (DMSO- $d_{6}, 75 \mathrm{MHz}$ ) $\delta 166.3,164.2$ (C-1, CO ester), 138.3, 136.5, 135.8, 130.3, 
$127.4,125.8,124.1,121.6\left(\mathrm{C}_{6} \mathrm{H}_{5}\right.$, thiophene $\left.\mathrm{C}\right)$, 108.6, $103.3(\mathrm{CH}=\mathrm{C}), 52.6\left(\mathrm{OCH}_{2} \mathrm{CH}_{3}\right), 50.4(\mathrm{C}-4), 36.3$ (C-5), $24.5\left(2 \mathrm{CH}_{3}\right), 16.2\left(\mathrm{OCH}_{2} \underline{\mathrm{CH}}_{3}\right)$; EIMS: $m / z 355[\mathrm{M}]^{+}$ (28\%); Anal. Calcd for $\mathrm{C}_{20} \mathrm{H}_{21} \mathrm{NO}_{3} \mathrm{~S}$ (355.45): C, 67.58; $\mathrm{H}$, $5.95 ;$ N, 3.94; S, 9.02\%. Found: C, 67.70; H, 5.64; N, 4.16; S, $8.86 \%$.

\section{2. 3. 2-Amino-4-(4-methoxybenzylidene)-7,7- dimethyl-5-oxo-4,5,6,7-tetrahydrobenzo $[b]$ thiophene-3-carbonitrile (5c)}

Yellow crystals from ethanol; m.p. $193-196^{\circ} \mathrm{C}$; yield $80 \%$. IR (KBr) cm $\mathrm{cm}^{-1}$ : 3473-3368 3055, 2985, 2220, 1688, 1630. ${ }^{1} \mathrm{H}$ NMR $\left(300 \mathrm{MHz}, \mathrm{DMSO}-d_{6}\right) \delta 7.46-7.21$ (m, $4 \mathrm{H}$, $\left.\mathrm{C}_{6} \mathrm{H}_{4}\right), 6.09(\mathrm{~s}, 1 \mathrm{H}, \mathrm{CH}), 4.36\left(\mathrm{~s}, 2 \mathrm{H}, \mathrm{D}_{2} \mathrm{O}\right.$ exchangeable, $\left.\mathrm{NH}_{2}\right), 3.64\left(\mathrm{~s}, 3 \mathrm{H}, \mathrm{OCH}_{3}\right), 2.36\left(\mathrm{~s}, 2 \mathrm{H}, \mathrm{CH}_{2}\right), 1.07,1.04(2 \mathrm{~s}$, $\left.6 \mathrm{H}, 2 \mathrm{CH}_{3}\right) ;{ }^{13} \mathrm{C}$ NMR (DMSO- $\left.d_{6}, 75 \mathrm{MHz}\right) \delta 166.3(\mathrm{C}-1)$, $138.2,136.4,135.6,130.9,128.5,126.3,123.8,120.8\left(\mathrm{C}_{6} \mathrm{H}_{5}\right.$, thiophene $\mathrm{C}), 116.8(\mathrm{CN}), 108.7,103.5(\mathrm{CH}=\mathrm{C}), 52.8$ $\left(\mathrm{OCH}_{3}\right), 50.6(\mathrm{C}-4), 36.5(\mathrm{C}-5), 24.6\left(2 \mathrm{CH}_{3}\right)$; EIMS: $m / z$ $338[\mathrm{M}]^{+}$(28\%); Anal. Calcd for $\mathrm{C}_{19} \mathrm{H}_{18} \mathrm{~N}_{2} \mathrm{O}_{2} \mathrm{~S}$ (338.42): C, 67.43; H, 5.36; N, 8.28; S, 9.47\%. Found: C, 67.72; H, 5.41; N, 8.16; S, 9.63\%.

\section{2. 4. Ethyl 2-Amino-4-(4-methoxybenzylidene)- 7,7-dimethyl-5-oxo-4,5,6,7- tetrahydrobenzo $[b]$ thiophene-3- carboxylate (5d)}

Orange crystals from ethanol; m.p. $188-190^{\circ} \mathrm{C}$; yield 67\%. IR (KBr) cm ${ }^{-1}: 4673,3359\left(\mathrm{NH}_{2}\right), 3055,2985,1689$, $1688,1630 .{ }^{1} \mathrm{H}$ NMR $\left(300 \mathrm{MHz}, \mathrm{DMSO}-d_{6}\right) \delta 7.46-7.24$ $\left(\mathrm{m}, 4 \mathrm{H}, \mathrm{C}_{6} \mathrm{H}_{4}\right), 6.03$ (s, 1H, CH), 4.38 (s, 2H, $\mathrm{D}_{2} \mathrm{O}$ exchangeable, $\mathrm{NH}_{2}$ ), $4.22\left(\mathrm{q}, 2 \mathrm{H}, J=7.19 \mathrm{~Hz}, \mathrm{OCH}_{2} \mathrm{CH}_{3}\right)$, $3.68\left(\mathrm{~s}, 3 \mathrm{H}, \mathrm{OCH}_{3}\right), 2.38\left(\mathrm{~s}, 2 \mathrm{H}, \mathrm{CH}_{2}\right), 1.13(\mathrm{t}, 3 \mathrm{H}, J=7.19$ $\left.\mathrm{Hz}, \mathrm{OCH}_{2} \mathrm{CH}_{3}\right), 1.08,1.01\left(2 \mathrm{~s}, 6 \mathrm{H}, 2 \mathrm{CH}_{3}\right) ;{ }^{13} \mathrm{C} \mathrm{NMR}$ (DMSO- $\left.d_{6}, 75 \mathrm{MHz}\right) \delta 166.6,164.8$ (C-1, CO ester), 138.3, $136.5,135.8,130.3,128.6,126.2,123.5,121.4\left(\mathrm{C}_{6} \mathrm{H}_{5}\right.$, thiophene $\mathrm{C}), 108.2,103.4(\mathrm{CH}=\mathrm{C}), 52.8\left(\mathrm{OCH}_{3}\right), 52.6$ $\left(\mathrm{OCH}_{2} \mathrm{CH}_{3}\right), 50.2(\mathrm{C}-4), 36.5(\mathrm{C}-5), 24.8\left(2 \mathrm{CH}_{3}\right), 16.2$ $\left(\mathrm{OCH}_{2} \underline{\mathrm{CH}}_{3}\right)$; EIMS: $m / z 385[\mathrm{M}]^{+}(32 \%)$; Anal. Calcd for $\mathrm{C}_{21} \mathrm{H}_{23} \mathrm{NO}_{4} \mathrm{~S}$ (385.48): C, 65.43; H, 6.01; N, 3.63; S, 8.32\%. Found: C, 65.59; H, 5.94; N, 3.80; S, 8.42\%.

\section{2. 5. 2-Amino-4-(4-chlorobenzylidene)-7,7- dimethyl-5-oxo-4,5,6,7-tetrahydrobenzo[b] thiophene-3-carbonitrile (5e)}

Yellow crystals from ethanol; m.p. $170-172{ }^{\circ} \mathrm{C}$; yield 75\%. IR (KBr) cm ${ }^{-1}$ : 3480-3359 $\left(\mathrm{NH}_{2}\right), 3055,2985,2220$, 1688, 1630. ${ }^{1} \mathrm{H}$ NMR (300 MHz, DMSO-d $\left.d_{6}\right) \delta 7.43-7.23$ $\left(\mathrm{m}, 4 \mathrm{H}, \mathrm{C}_{6} \mathrm{H}_{4}\right), 6.05(\mathrm{~s}, 1 \mathrm{H}, \mathrm{CH}), 4.34\left(\mathrm{~s}, 2 \mathrm{H}, \mathrm{D}_{2} \mathrm{O}\right.$ exchangeable, $\left.\mathrm{NH}_{2}\right), 2.38\left(\mathrm{~s}, 2 \mathrm{H}, \mathrm{CH}_{2}\right), 1.07,1.04(2 \mathrm{~s}, 6 \mathrm{H}$, $2 \mathrm{CH}_{3}$ ); ${ }^{13} \mathrm{C}$ NMR (DMSO- $\left.d_{6}, 75 \mathrm{MHz}\right) \delta 166.6$ (C-1), $138.3,135.9,134.6,130.3,128.2,126.7,123.5,120.4\left(\mathrm{C}_{6} \mathrm{H}_{5}\right.$, thiophene C), $116.9(\mathrm{CN}), 108.3,103.2(\mathrm{CH}=\mathrm{C}), 50.3$
(C-4), 36.2 (C-5), $24.7\left(2 \mathrm{CH}_{3}\right)$; EIMS: $m / z 342[\mathrm{M}]^{+}(35 \%)$; Anal. Calcd for $\mathrm{C}_{18} \mathrm{H}_{15} \mathrm{ClN}_{2} \mathrm{OS}$ (342.84): C, 63.06; $\mathrm{H}, 4.41$ N, 8.17; S, 9.35\%. Found: C, 63.29; H, 4.69; N, 8.31; S, $9.42 \%$.

\section{2. 6. Ethyl 2-amino-4-(4-chlorobenzylidene)- 7,7-dimethyl-5-oxo-4,5,6,7- tetrahydrobenzo $[b]$ thiophene-3- carboxylate $(5 f)$}

Yellow crystals from ethanol; m.p. $126-128^{\circ} \mathrm{C}$; yield $76 \%$. IR (KBr) cm $\mathrm{cm}^{-1}$ : 4673, $3368\left(\mathrm{NH}_{2}\right), 3055,2983,1703$, $1688,1630 .{ }^{1} \mathrm{H}$ NMR $\left(300 \mathrm{MHz}, \mathrm{DMSO}-d_{6}\right) \delta 7.46-7.23$ $\left(\mathrm{m}, 4 \mathrm{H}, \mathrm{C}_{6} \mathrm{H}_{5}\right), 6.02$ (s, 1H, CH), 4.39 (s, 2H, $\mathrm{D}_{2} \mathrm{O}$ exchangeable, $\left.\mathrm{NH}_{2}\right), 4.23\left(\mathrm{q}, 2 \mathrm{H}, J=6.72 \mathrm{~Hz}, \mathrm{OCH}_{2} \mathrm{CH}_{3}\right)$, $2.35\left(\mathrm{~s}, 2 \mathrm{H}, \mathrm{CH}_{2}\right), 1.13\left(\mathrm{t}, 3 \mathrm{H}, J=6.72 \mathrm{~Hz}, \mathrm{OCH}_{2} \underline{\mathrm{CH}}_{3}\right)$, $1.09,1.053\left(2 \mathrm{~s}, 6 \mathrm{H}, 2 \mathrm{CH}_{3}\right) ;{ }^{13} \mathrm{C} \mathrm{NMR}$ (DMSO- $d_{6}, 75 \mathrm{MHz}$ ) $\delta$ 166.6, 164.5 (C-1, CO ester), 138.2, 136.3, 135.6, 130.4, $127.3,125.2,124.6,121.3\left(\mathrm{C}_{6} \mathrm{H}_{5}\right.$, thiophene $\left.\mathrm{C}\right), 108.2$, $103.5(\mathrm{CH}=\mathrm{C}), 52.4\left(\mathrm{OCH}_{2} \mathrm{CH}_{3}\right), 50.6(\mathrm{C}-4), 36.8(\mathrm{C}-5)$, $24.5\left(2 \mathrm{CH}_{3}\right), 16.2\left(\mathrm{OCH}_{2} \mathrm{CH}_{3}\right)$; EIMS: $m / z 389[\mathrm{M}]^{+}$ (32\%); Anal. Calcd for $\mathrm{C}_{20} \mathrm{H}_{20} \mathrm{ClNO}_{3} \mathrm{~S}$ (389.90): C, 61.61; H, 5.17; N, 3.59; S, 8.22\%. Found: C, 61.49; H, 5.36; N, $3.69 ; \mathrm{S}, 8.38 \%$.

\section{2. 7. General Procedure for the Synthesis of the 4,5,6,7-Tetrahydro- $2 \mathrm{H}$-indazole Derivatives $7 \mathbf{a}-\mathbf{f}$}

To a solution of any of $3 \mathbf{a}(2.28 \mathrm{~g}, 0.01 \mathrm{~mol}), 3 \mathbf{b}(2.58$ $\mathrm{g}, 0.01 \mathrm{~mol})$ or $3 \mathrm{c}(2.62 \mathrm{~g}, 0.01 \mathrm{~mol})$ in ethanol $(40 \mathrm{~mL})$ either of hydrazine hydrate $(0.1 \mathrm{~mL}, 0.02 \mathrm{~mol})$ or phenylhydrazine $(3.16 \mathrm{~g}, 0.02 \mathrm{~mol}$ ) was added. The reaction mixture, in each case, was heated under reflux for $3 \mathrm{~h}$ then was left to cool and the formed solid product was collected by filtration upon pouring onto ice/water mixture containing a few drops of hydrochloric acid.

\section{2. 7. 1. 4-Hydrazono-6,6-dimethyl-3-phenyl-4,5,6,7- tetrahydro- $2 \mathrm{H}$-indazole $(7 \mathrm{a})$}

Pale yellow crystals from ethanol; m.p. $175-177^{\circ} \mathrm{C}$; yield $68 \%$. IR $(\mathrm{KBr}) \mathrm{cm}^{-1}: 3497-3336\left(\mathrm{NH}, \mathrm{NH}_{2}\right), 3055$, 2985, 1663, 1632. ${ }^{1} \mathrm{H}$ NMR (300 MHz, DMSO- $\left.d_{6}\right) \delta 8.26$ (s, $1 \mathrm{H}, \mathrm{D}_{2} \mathrm{O}$ exchangeable, $\left.\mathrm{NH}\right), 7.43-7.26\left(\mathrm{~m}, 5 \mathrm{H}, \mathrm{C}_{6} \mathrm{H}_{5}\right)$, $5.29\left(\mathrm{~s}, 2 \mathrm{H}, \mathrm{D}_{2} \mathrm{O}\right.$ exchangeable, $\left.\mathrm{NH}_{2}\right), 2.41,2.38(2 \mathrm{~s}, 4 \mathrm{H}$, $\left.2 \mathrm{CH}_{2}\right), 1.07,1.06\left(2 \mathrm{~s}, 6 \mathrm{H}, 2 \mathrm{CH}_{3}\right) ;{ }^{13} \mathrm{C}$ NMR (DMSO- $d_{6}, 75$ $\mathrm{MHz}) \delta 172.3(\mathrm{C}-4), 140.6,138.3,127.8,125.4,124.3$, $121.0\left(\mathrm{C}_{6} \mathrm{H}_{5}, \mathrm{C}-3, \mathrm{C}-4\right), 34.6$ (C-6), 36.8, 24.9, (C-7, C-5) $24.3\left(2 \mathrm{CH}_{3}\right)$; EIMS: $m / z 254[\mathrm{M}]^{+}(28 \%)$; Anal. Calcd for $\mathrm{C}_{15} \mathrm{H}_{18} \mathrm{~N}_{4}$ (254.33): C, 70.84; H, 7.13; N, 22.03\%. Found: C, 70.69; H, 6.93; N, 21.79\%.

\section{2. 7. 2. 4-Hydrazono-3-(4-methoxyphenyl)-6,6- dimethyl-4,5,6,7-tetrahydro- $2 \mathrm{H}$-indazole $(7 \mathrm{~b})$ \\ Orange crystals from ethanol; m.p. $189-192{ }^{\circ} \mathrm{C}$; yield $70 \%$. IR $(\mathrm{KBr}) \mathrm{cm}^{-1}: 3474-3353\left(\mathrm{NH}, \mathrm{NH}_{2}\right), 3055$,}


$2985,1660,1630 .{ }^{1} \mathrm{H}$ NMR $\left(300 \mathrm{MHz}, \mathrm{DMSO}-d_{6}\right) \delta 8.28$ (s, $1 \mathrm{H}, \mathrm{D}_{2} \mathrm{O}$ exchangeable, $\left.\mathrm{NH}\right), 7.48-7.23\left(\mathrm{~m}, 4 \mathrm{H}, \mathrm{C}_{6} \mathrm{H}_{4}\right)$, $5.27\left(\mathrm{~s}, 2 \mathrm{H}, \mathrm{D}_{2} \mathrm{O}\right.$ exchangeable, $\left.\mathrm{NH}_{2}\right), 3.52\left(\mathrm{~s}, 3 \mathrm{H}, \mathrm{OCH}_{3}\right)$, 2.46, $2.32\left(2 \mathrm{~s}, 4 \mathrm{H}, 2 \mathrm{CH}_{2}\right), 1.09,1.06\left(2 \mathrm{~s}, 6 \mathrm{H}, 2 \mathrm{CH}_{3}\right) ;{ }^{13} \mathrm{C}$ NMR (DMSO- $\left.d_{6}, 75 \mathrm{MHz}\right) \delta 172.6$ (C-4), 140.3, 138.6, 127.4, 126.6, 123.1, $120.8\left(\mathrm{C}_{6} \mathrm{H}_{5}, \mathrm{C}-3, \mathrm{C}-4\right), 50.2\left(\mathrm{OCH}_{3}\right)$, 34.8 (C-6), 36.6, 24.8, (C-7, C-5) $24.1\left(2 \mathrm{CH}_{3}\right)$; EIMS: $m / z$ $284[\mathrm{M}]^{+}(30 \%)$; Anal. Calcd for $\mathrm{C}_{16} \mathrm{H}_{20} \mathrm{~N}_{4} \mathrm{O}$ (284.36): C, $67.58 ; \mathrm{H}, 7.09 ; \mathrm{N}, 19.70 \%$. Found: C, 67.39; H, 6.84; N, $19.66 \%$

\section{2. 7.3.3-(4-Chlorophenyl)-4-hydrazono-6,6-} dimethyl-4,5,6,7-tetrahydro- $2 \mathrm{H}$-indazole $(7 \mathrm{c})$

Yellow crystals from 1,4-dioxan; m.p. $185-187^{\circ} \mathrm{C}$; yield $77 \%$. IR $(\mathrm{KBr}) \mathrm{cm}^{-1}: 3493-3342\left(\mathrm{NH}, \mathrm{NH}_{2}\right), 3057$, $2985,1662,1630 .{ }^{1} \mathrm{H}$ NMR $\left(300 \mathrm{MHz}, \mathrm{DMSO}-d_{6}\right) \delta 8.27$ (s, $1 \mathrm{H}, \mathrm{D}_{2} \mathrm{O}$ exchangeable, $\left.\mathrm{NH}\right), 7.52-7.25\left(\mathrm{~m}, 4 \mathrm{H}, \mathrm{C}_{6} \mathrm{H}_{4}\right)$, $5.25\left(\mathrm{~s}, 2 \mathrm{H}, \mathrm{D}_{2} \mathrm{O}\right.$ exchangeable, $\left.\mathrm{NH}_{2}\right), 2.43,2.36(2 \mathrm{~s}, 4 \mathrm{H}$, $\left.2 \mathrm{CH}_{2}\right), 1.08,1.03\left(2 \mathrm{~s}, 6 \mathrm{H}, 2 \mathrm{CH}_{3}\right) ;{ }^{13} \mathrm{C}$ NMR (DMSO- $d_{6}, 75$ $\mathrm{MHz}) \delta 172.8(\mathrm{C}-4), 140.1,138.4,128.3,125.5,122.6$, $120.2\left(\mathrm{C}_{6} \mathrm{H}_{5}, \mathrm{C}-3, \mathrm{C}-4\right), 34.6$ (C-6), 36.3, 24.5, (C-7, C-5) $24.3\left(2 \mathrm{CH}_{3}\right)$; EIMS: $m / z 288[\mathrm{M}]^{+}(24 \%)$; Anal. Calcd for $\mathrm{C}_{15} \mathrm{H}_{17} \mathrm{ClN}_{4}$ (288.78): C, 62.39; H, 5.93; N, 19.40\%. Found: C, 62.58; H, 6.15; N, 19.52\%.

\section{2. 7. 4. 6,6-Dimethyl-2,3-diphenyl-4-(2- phenylhydrazono)-4,5,6,7-tetrahydro- $2 \mathrm{H}$ - indazole $(7 \mathrm{~d})$}

Pale yellow crystals from ethanol; m.p. $171-173^{\circ} \mathrm{C}$; yield $60 \%$. IR $(\mathrm{KBr}) \mathrm{cm}^{-1}: 3482-3346\left(\mathrm{NH}, \mathrm{NH}_{2}\right), 3055$, $2985,1665,1632 .{ }^{1} \mathrm{H}$ NMR $\left(300 \mathrm{MHz}, \mathrm{DMSO}-d_{6}\right) \delta 8.28$ (s, $1 \mathrm{H}, \mathrm{D}_{2} \mathrm{O}$ exchangeable, $\left.\mathrm{NH}\right), 7.46-7.22(\mathrm{~m}, 15 \mathrm{H}$, $\left.3 \mathrm{C}_{6} \mathrm{H}_{5}\right), 2.42,2.38\left(2 \mathrm{~s}, 4 \mathrm{H}, 2 \mathrm{CH}_{2}\right), 1.09,1.07(2 \mathrm{~s}, 6 \mathrm{H}$, $2 \mathrm{CH}_{3}$ ); ${ }^{13} \mathrm{C}$ NMR (DMSO- $\left.d_{6}, 75 \mathrm{MHz}\right) \delta 172.6(\mathrm{C}-4)$, $140.4,138.1,129.6,129.3,128.5,127.8,127.4,126.1$, $126.5,126.9,125.6,125.2,124.0,121.2\left(3 \mathrm{C}_{6} \mathrm{H}_{5}, \mathrm{C}-3, \mathrm{C}-4\right)$, 34.8 (C-6), 36.4, 24.7 (C-7, C-5) $24.5\left(2 \mathrm{CH}_{3}\right)$; EIMS: $\mathrm{m} / z$ $406[\mathrm{M}]^{+}(24 \%)$; Anal. Calcd for $\mathrm{C}_{27} \mathrm{H}_{26} \mathrm{~N}_{4}$ (406.52): C, 79.77; H, 6.45; N, 13.78\%. Found: C, 79.84; H, 6.62; N, $13.53 \%$.

\section{2. 7.5.3-(4-Methoxyphenyl)-6,6-dimethyl-2-phenyl- 4-(2-phenylhydrazono)-4,5,6,7-tetrahydro- $2 \mathrm{H}$-indazolele $(7 \mathrm{e})$}

Orange crystals from ethanol; m.p. $128-130{ }^{\circ} \mathrm{C}$; yield 76\%. IR (KBr) cm ${ }^{-1}: 3486-3336(\mathrm{NH}), 3055,2985,1660$, 1630. ${ }^{1} \mathrm{H}$ NMR $\left(300 \mathrm{MHz}, \mathrm{DMSO}-d_{6}\right) \delta 8.31\left(\mathrm{~s}, 1 \mathrm{H}, \mathrm{D}_{2} \mathrm{O}\right.$ exchangeable, $\mathrm{NH}), 7.53-7.26\left(\mathrm{~m}, 14 \mathrm{H}, \mathrm{C}_{6} \mathrm{H}_{5}, 2 \mathrm{C}_{6} \mathrm{H}_{4}\right)$, $3.61\left(\mathrm{~s}, 3 \mathrm{H}, \mathrm{OCH}_{3}\right), 2.48,2.36\left(2 \mathrm{~s}, 4 \mathrm{H}, 2 \mathrm{CH}_{2}\right), 1.08,1.07$ $\left(2 \mathrm{~s}, 6 \mathrm{H}, 2 \mathrm{CH}_{3}\right.$ ); ${ }^{13} \mathrm{C}$ NMR (DMSO- $\left.d_{6}, 75 \mathrm{MHz}\right) \delta 172.8$ (C-4), 140.7, 138.3, 128.8, 129.3, 128.5, 127.8, 127.3, 126.1, $126.8,125.9,125.6,124.3,123.7,120.2\left(2 \mathrm{C}_{6} \mathrm{H}_{5}, \mathrm{C}_{6} \mathrm{H}_{4}, \mathrm{C}-3\right.$, C-4), $50.2\left(\mathrm{OCH}_{3}\right), 34.1$ (C-6), 36.6, 24.3 (C-7, C-5) 24.2 $\left(2 \mathrm{CH}_{3}\right)$; EIMS: $m / z 436[\mathrm{M}]^{+}(38 \%)$; Anal. Calcd for $\mathrm{C}_{28} \mathrm{H}_{28} \mathrm{~N}_{4} \mathrm{O}$ (436.55): C, 77.04; H, 6.46; N, 12.83\%. Found: C, 77.25; H, 6.58; N, 12.64\%.
2. 2. 7. 6. 3-(4-Chlorophenyl)-6,6-dimethyl-2-phenyl-4(2-phenylhydrazono)-4,5,6,7-tetrahydro- $2 \mathrm{H}$ indazole $(7 \mathbf{f})$

Pale yellow crystals from ethanol; m.p. $126-128^{\circ} \mathrm{C}$; yield $66 \%$. IR $(\mathrm{KBr}) \mathrm{cm}^{-1}: 3492-3327(\mathrm{NH}), 3055,2985$, $1660,1630 .{ }^{1} \mathrm{H}$ NMR $\left(300 \mathrm{MHz}, \mathrm{DMSO}-d_{6}\right) \delta 8.26(\mathrm{~s}, 1 \mathrm{H}$, $\mathrm{D}_{2} \mathrm{O}$ exchangeable, $\left.\mathrm{NH}\right), 7.47-7.21\left(\mathrm{~m}, 14 \mathrm{H}, 2 \mathrm{C}_{6} \mathrm{H}_{5}\right.$, $\left.\mathrm{C}_{6} \mathrm{H}_{4}\right), 2.46,2.38\left(2 \mathrm{~s}, 4 \mathrm{H}, 2 \mathrm{CH}_{2}\right), 1.08,1.04\left(2 \mathrm{~s}, 6 \mathrm{H}, 2 \mathrm{CH}_{3}\right)$; ${ }^{13} \mathrm{C}$ NMR (DMSO- $\left.d_{6}, 75 \mathrm{MHz}\right) \delta 172.4(\mathrm{C}-4), 140.1,138.6$, $129.3,129.1,128.4,127.5,127.4,126.3,126.5,126.7,125.4$, 125.2, 124.0, $121.0\left(2 \mathrm{C}_{6} \mathrm{H}_{5}, \mathrm{C}_{6} \mathrm{H}_{4}, \mathrm{C}-3, \mathrm{C}-4\right), 34.5$ (C-6), 36.4, 24.7 (C-7, C-5) $24.8\left(2 \mathrm{CH}_{3}\right)$; EIMS: $m / z 440[\mathrm{M}]^{+}$ (36\%); Anal. Calcd for $\mathrm{C}_{27} \mathrm{H}_{25} \mathrm{ClN}_{4}$ (440.97): C, 73.54; $\mathrm{H}$, $5.71 ; \mathrm{N}, 12.71 \%$. Found: C, 73.58; H, 5.68; N, 12.86\%.

\section{2. 8. General Procedure for the Synthesis of the Dihydrobenzo $[c]$ isoxazole Derivatives 9a-c}

To a solution of any of $3 \mathbf{a}(2.28 \mathrm{~g}, 0.01 \mathrm{~mol}), \mathbf{3 b}(2.58$ $\mathrm{g}, 0.01 \mathrm{~mol})$ or $3 \mathrm{c}(2.62 \mathrm{~g}, 0.01 \mathrm{~mol})$ in ethanol $(40 \mathrm{~mL})$ containing sodium acetate $(1.00 \mathrm{~g})$ hydroxylamine hydrochloride $(1.40 \mathrm{~g}, 0.02 \mathrm{~mol})$ was added. The reaction mixture was heated under reflux for $2 \mathrm{~h}$ then poured onto ice/water and the formed solid product was collected by filtration.

\section{2. 8. 1. 6,6-Dimethyl-3-phenyl-6,7-dihydrobenzo[c] isoxazol-4 $(5 H)$-one oxime $(9 a)$}

Pale yellow crystals from 1,4-dioxan; m.p. 183-185 ${ }^{\circ} \mathrm{C}$; yield 68\%. IR (KBr) cm cm $^{-1}$ 3572-3326 $(\mathrm{OH}), 3055$, 2985, 1663, 1631. ${ }^{1} \mathrm{H}$ NMR $\left(300 \mathrm{MHz}, \mathrm{DMSO}-d_{6}\right) \delta 9.52$ (s, $1 \mathrm{H}, \mathrm{D}_{2} \mathrm{O}$ exchangeable, $\left.\mathrm{OH}\right), 7.42-7.26\left(\mathrm{~m}, 5 \mathrm{H}, \mathrm{C}_{6} \mathrm{H}_{5}\right)$, 2.47, $2.33\left(2 \mathrm{~s}, 4 \mathrm{H}, 2 \mathrm{CH}_{2}\right), 1.08,1.05\left(2 \mathrm{~s}, 6 \mathrm{H}, 2 \mathrm{CH}_{3}\right) ;{ }^{13} \mathrm{C}$ NMR (DMSO- $\left.d_{6}, 75 \mathrm{MHz}\right) \delta 175.3(\mathrm{C}-4), 140.1,137.6$, 127.8, 126.5, 124.0, $121.2\left(\mathrm{C}_{6} \mathrm{H}_{5}, \mathrm{C}-3, \mathrm{C}-4\right), 34.3$ (C-6), 36.8, 24.5 (C-7, C-5), $24.8\left(2 \mathrm{CH}_{3}\right)$; EIMS: $m / z 256[\mathrm{M}]^{+}$ (30\%); Anal. Calcd for $\mathrm{C}_{15} \mathrm{H}_{16} \mathrm{~N}_{2} \mathrm{O}_{2}$ (256.30): C, 70.29; $\mathrm{H}$, 6.29 ; N, 10.93\%. Found: C, 70.31; H, 6.37; N, 11.25\%.

\section{2. 8. 2. 3-(4-Methoxyphenyl)-6,6-dimethyl-6,7- \\ dihydrobenzo $[c]$ isoxazol- $4(5 H)$-one oxime $(9 b)$}

Pale yellow crystals from ethanol; m.p. $177-180^{\circ} \mathrm{C}$; yield $58 \%$. IR $(\mathrm{KBr}) \mathrm{cm}^{-1}$ : 3529-3336 (OH), 3055, 2985, $1663,1631 .{ }^{1} \mathrm{H}$ NMR $\left(300 \mathrm{MHz}, \mathrm{DMSO}-d_{6}\right) \delta 9.40(\mathrm{~s}, 1 \mathrm{H}$, $\mathrm{D}_{2} \mathrm{O}$ exchangeable, $\left.\mathrm{OH}\right), 7.48-7.26\left(\mathrm{~m}, 4 \mathrm{H}, \mathrm{C}_{6} \mathrm{H}_{4}\right), 3.52(\mathrm{~s}$, $\left.3 \mathrm{H}, \mathrm{OCH}_{3}\right), 2.42,2.35\left(2 \mathrm{~s}, 4 \mathrm{H}, 2 \mathrm{CH}_{2}\right), 1.09,1.04(2 \mathrm{~s}, 6 \mathrm{H}$, $\left.2 \mathrm{CH}_{3}\right) ;{ }^{13} \mathrm{C}$ NMR (DMSO- $\left.d_{6}, 75 \mathrm{MHz}\right) \delta 172.8(\mathrm{C}-4)$, $140.7,138.1,126.9,125.6,125.6,120.8\left(\mathrm{C}_{6} \mathrm{H}_{4}, \mathrm{C}-3, \mathrm{C}-4\right)$, $50.8\left(\mathrm{OCH}_{3}\right), 34.4(\mathrm{C}-6), 36.9,24.3(\mathrm{C}-7, \mathrm{C}-5) 24.8\left(2 \mathrm{CH}_{3}\right)$; EIMS: $m / z 286[\mathrm{M}]^{+}$(30\%); Anal. Calcd for $\mathrm{C}_{16} \mathrm{H}_{18} \mathrm{~N}_{2} \mathrm{O}_{3}$ (286.33): C, 67.12; H, 6.34; N, 9.78\%. Found: C, 67.26; H, $6.26 ; \mathrm{N}, 9.80 \%$.

\section{2. 8. 3. 3-(4-Chlorophenyl)-6,6-dimethyl-6,7- \\ dihydrobenzo $[c]$ isoxazol- $4(5 H)$-one oxime $(9 \mathrm{c})$ \\ Pale yellow crystals from 1,4-dioxan; m.p. 201-203 \\ ${ }^{\circ} \mathrm{C}$; yield 77\%. IR (KBr) cm ${ }^{-1}$ : 3552-3361 (OH), 3055,}


$2985,1664,1630 .{ }^{1} \mathrm{H}$ NMR $\left(300 \mathrm{MHz}, \mathrm{DMSO}-d_{6}\right) \delta 9.53$ (s, $1 \mathrm{H}, \mathrm{D}_{2} \mathrm{O}$ exchangeable, $\left.\mathrm{OH}\right), 7.46-7.23(\mathrm{~m}, 4 \mathrm{H}$, $\left.\mathrm{C}_{6} \mathrm{H}_{4}\right), 2.49,2.31\left(2 \mathrm{~s}, 4 \mathrm{H}, 2 \mathrm{CH}_{2}\right), 1.06,1.02(2 \mathrm{~s}, 6 \mathrm{H}$, $\left.2 \mathrm{CH}_{3}\right) ;{ }^{13} \mathrm{C}$ NMR (DMSO- $\left.d_{6}, 75 \mathrm{MHz}\right) \delta 175.5(\mathrm{C}-4)$, $140.3,137.2,127.6,125.8,123.3,120.9\left(\mathrm{C}_{6} \mathrm{H}_{5}, \mathrm{C}-3, \mathrm{C}-4\right)$, 34.1 (C-6), 36.6, 24.2 (C-7, C-5), $24.3\left(2 \mathrm{CH}_{3}\right)$; EIMS: $\mathrm{m} / z$ $290[\mathrm{M}]^{+}$(26\%); Anal. Calcd for $\mathrm{C}_{15} \mathrm{H}_{15} \mathrm{ClN}_{2} \mathrm{O}_{2}$ (290.74): C, 61.97; H, 5.20; N, 9.64\%. Found: C, 62.79; H, 4.86; N, $9.80 \%$.

\section{2. 9. General Procedure of the Synthesis of the Hydrazone Derivatives 11a,b}

To a cold solution $\left(0-5^{\circ} \mathrm{C}\right)$ of compound $\mathbf{1}(1.40 \mathrm{~g}$, $0.01 \mathrm{~mol})$ in ethanol $(40 \mathrm{~mL})$ containing sodium acetate $(3.0 \mathrm{~g})$ either 4-methylnaphthalen-1-diazonium salt $(0.01$ mol) or 4-chloronaphthalen-1-diazonium salt [prepared by the addition of sodium nitrite solution $(0.70 \mathrm{~g}, 0.01$ mol) to a cold solution $\left(0-5^{\circ} \mathrm{C}\right)$ of either of 4-methylnaphthalen-1-amine (1.57 g, $0.01 \mathrm{~mol})$ or 4-chloromethylnaphthalen-1-amine $(1.77 \mathrm{~g}, 0.01 \mathrm{~mol})$ with continuous stirring] was added with continuous stirring. The reaction mixture, in each case was stirred at room temperature for an additional $2 \mathrm{~h}$ and the formed solid product was collected by filtration.

\section{2. 9. 1. 5,5-Dimethyl-2-(2-(4-methylnaphthalen-1- yl)hydrazono)cyclohexane-1,3-dione (11a)}

Pale yellow crystals from ethanol; m.p. $220-223^{\circ} \mathrm{C}$; yield $68 \%$. IR $(\mathrm{KBr}) \mathrm{cm}^{-1}: 3468-3334(\mathrm{NH}), 3055,2985$, $1689,1687,1663,1630 .{ }^{1} \mathrm{H}$ NMR $\left(300 \mathrm{MHz}\right.$, DMSO- $\left.d_{6}\right) \delta$ $8.29\left(\mathrm{~s}, 1 \mathrm{H}, \mathrm{D}_{2} \mathrm{O}\right.$ exchangeable, $\left.\mathrm{NH}\right), 7.48-7.23(\mathrm{~m}, 6 \mathrm{H}$, naphthalene $\mathrm{H}), 2.41,2.38\left(2 \mathrm{~s}, 4 \mathrm{H}, 2 \mathrm{CH}_{2}\right), 2.89(\mathrm{~s}, 3 \mathrm{H}$, $\left.\mathrm{CH}_{3}\right), 1.07,1.06\left(2 \mathrm{~s}, 6 \mathrm{H}, 2 \mathrm{CH}_{3}\right) ;{ }^{13} \mathrm{C}$ NMR (DMSO- $d_{6}, 75$ MHz) $\delta 172.8$ (C-2), 166.2, 164.8 (C-1, C-3), 138.6, 135.2, $133.1,129.6,126.5,126.1,124.7,123.9,122.3,120.6$ (naphthalene C), $34.8(\mathrm{C}-6), 36.5,32.8\left(\mathrm{CH}_{3}\right), 24.3(\mathrm{C}-7, \mathrm{C}-5)$ $24.5\left(2 \mathrm{CH}_{3}\right)$; EIMS: $m / z 308[\mathrm{M}]^{+}(21 \%)$; Anal. Calcd for $\mathrm{C}_{19} \mathrm{H}_{20} \mathrm{~N}_{2} \mathrm{O}_{2}$ (308.37): C, 74.00; H, 6.54; N, 9.08\%. Found: C, 73.96; H, 6.82; N, 8.79\%.

\section{2. 9. 2. 2-(2-(4-Chloronaphthalen-1-yl)hydrazono)-}

5,5-dimethylcyclohexane-1,3-dione (11b)

Pale yellow crystals from ethanol; m.p. $211-213^{\circ} \mathrm{C}$; yield $74 \%$. IR $(\mathrm{KBr}) \mathrm{cm}^{-1}: 3493-3358(\mathrm{NH}), 3055,2985$, $1688,1687,1663,1630 .{ }^{1} \mathrm{H}$ NMR $\left(300 \mathrm{MHz}, \mathrm{DMSO}-d_{6}\right)$ $\delta 8.32\left(\mathrm{~s}, 1 \mathrm{H}, \mathrm{D}_{2} \mathrm{O}\right.$ exchangeable, $\left.\mathrm{NH}\right), 7.56-7.25(\mathrm{~m}, 6 \mathrm{H}$, naphthalene $\mathrm{H}), 2.46,2.33\left(2 \mathrm{~s}, 4 \mathrm{H}, 2 \mathrm{CH}_{2}\right), 1.08,1.06(2 \mathrm{~s}$, $\left.6 \mathrm{H}, 2 \mathrm{CH}_{3}\right) ;{ }^{13} \mathrm{C}$ NMR (DMSO- $\left.d_{6}, 75 \mathrm{MHz}\right) \delta 172.9$ (C-2), 166.7, 164.8 (C-1, C-3), 138.8, 135.6, 134.41, $129.2,127.8,125.3,124.2,123.7,122 . .1,120.4$ (naphthalene C), 34.8 (C-6), 36.8, 24.3 (C-7, C-5) $24.2\left(2 \mathrm{CH}_{3}\right)$; EIMS: $m / z \quad 328 \quad[\mathrm{M}]^{+} \quad(18 \%)$; Anal. Calcd for $\mathrm{C}_{18} \mathrm{H}_{17} \mathrm{ClN}_{2} \mathrm{O}_{2}$ (328.79): C, 65.75; H, 5.21; N, 8.52\%. Found: C, 65.49; H, 5.06; N, 8.63\%.

\section{2. 10. General Procedure for the Synthesis of the 6,7-Dihydrobenzo[b] thiophene Derivatives 12a-d}

To a solution of either of $11 \mathrm{a}(3.08 \mathrm{~g}, 0.01 \mathrm{~mol}), \mathbf{1 1 b}$ $(3.28 \mathrm{~g}, 0.01 \mathrm{~mol})$ in 1,4-dioxan $(40 \mathrm{~mL})$ containing triethylamine $(0.50 \mathrm{~mL})$ each of elemental sulfur $(0.32 \mathrm{~g}, 0.01$ $\mathrm{mol}$ ) and either of malononitrile $(0.66 \mathrm{~g}, 0.01 \mathrm{~mol})$ or ethyl cyanoacetate $(1.07 \mathrm{~g}, 0.01 \mathrm{~mol})$ were added. The reaction mixture was heated under reflux for $1 \mathrm{~h}$ then left to cool. The formed solid product was collected by filtration, dried and crystallized from ethanol to give 12a-d, respectively.

\section{2 .10. 1. 2-Amino-7,7-dimethyl-4-(2-(4- methylnaphthalen -1-yl)hydrazono)-5-oxo- 4,5,6,7-tetrahydrobenzo $[b]$ thiophene-3- carbonitrile (12a)}

Pale yellow crystals from ethanol; m.p. $218-220^{\circ} \mathrm{C}$; yield $68 \%$. IR $(\mathrm{KBr}) \mathrm{cm}^{-1}: 3480-3350\left(\mathrm{NH}, \mathrm{NH}_{2}\right), 3055$, $2985,2220,1688,1663,1630 .{ }^{1} \mathrm{H}$ NMR $(300 \mathrm{MHz}, \mathrm{DM}-$ SO- $\left.d_{6}\right) \delta 8.37$ (s, $1 \mathrm{H}, \mathrm{D}_{2} \mathrm{O}$ exchangeable, $\left.\mathrm{NH}\right), 7.48-7.23$ $(\mathrm{m}, 6 \mathrm{H}$, naphthalene $\mathrm{H}), 4.85\left(\mathrm{~s}, 2 \mathrm{H}, \mathrm{D}_{2} \mathrm{O}\right.$ exchangeable, $\mathrm{NH}_{2}$ ), 2.86 (s, $\left.3 \mathrm{H}, \mathrm{CH}_{3}\right), 2.42\left(\mathrm{~s}, 2 \mathrm{H}, \mathrm{CH}_{2}\right), 1.09,1.06$ (2s, $\left.6 \mathrm{H}, 2 \mathrm{CH}_{3}\right) ;{ }^{13} \mathrm{C}$ NMR (DMSO- $\left.d_{6}, 75 \mathrm{MHz}\right) \delta 175.7(\mathrm{C}-2)$, 166.2, (C-3), 138.8, 136.6, 133.8, 129.3, 126.2, 126.4, 124.2, $123.3,122.8,120.2$ (naphthalene C), $116.8(\mathrm{CN}), 34.6$ (C-6), $32.3\left(\mathrm{CH}_{3}\right), 24.1$ (C-7, C-5), $24.8\left(2 \mathrm{CH}_{3}\right)$; EIMS: $\mathrm{m} / z$ $388[\mathrm{M}]^{+}(28 \%)$; Anal. Calcd for $\mathrm{C}_{22} \mathrm{H}_{20} \mathrm{~N}_{4} \mathrm{OS}$ (388.49): C, $68.02 ; \mathrm{H}, 5.19 ; \mathrm{N}, 14.42 ; \mathrm{S}, 8.25 \%$. Found: C, 67.83; H, 5.27; N, 8.39; S, 8.36\%.

\section{2. 10. 2. Ethyl 2-Amino-7,7-dimethyl-4-(2-(4- methylnaphthalen-1-yl)hydrazono)-5-oxo- 4,5,6,7-tetrahydrobenzo[b] thiophene-3- carboxylate (12b)}

Pale yellow crystals from ethanol; m.p. $177-179{ }^{\circ} \mathrm{C}$; yield 73\%. IR ( $\mathrm{KBr}) \mathrm{cm}^{-1}: 3468-3342\left(\mathrm{NH}, \mathrm{NH}_{2}\right), 3055$, 2985, 1689, 1687, 1663, 1630. ${ }^{1} \mathrm{H}$ NMR $(300 \mathrm{MHz}, \mathrm{DM}-$ SO- $\left.d_{6}\right) \delta 8.39\left(\mathrm{~s}, 1 \mathrm{H}, \mathrm{D}_{2} \mathrm{O}\right.$ exchangeable, $\left.\mathrm{NH}\right), 7.56-7.25(\mathrm{~m}$, $6 \mathrm{H}$, naphthalene $\mathrm{H}), 4.88$ (s, $2 \mathrm{H}, \mathrm{D}_{2} \mathrm{O}$ exchangeable, $\mathrm{NH}_{2}$ ), $4.21\left(\mathrm{q}, 2 \mathrm{H}, J=6.72 \mathrm{~Hz}, \mathrm{OCH}_{2} \mathrm{CH}_{3}\right), 2.86\left(\mathrm{~s}, 3 \mathrm{H}, \mathrm{CH}_{3}\right), 2.41$ $\left(\mathrm{s}, 2 \mathrm{H}, \mathrm{CH}_{2}\right), 1.13\left(\mathrm{t}, 3 \mathrm{H}, J=6.72 \mathrm{~Hz}, \mathrm{OCH}_{2} \mathrm{CH}_{3}\right), 1.09,1.07$ $\left(2 \mathrm{~s}, 6 \mathrm{H}, 2 \mathrm{CH}_{3}\right) ;{ }^{13} \mathrm{C}$ NMR (DMSO- $\left.d_{6}, 75 \mathrm{MHz}\right) \delta 175.8(\mathrm{C}-$ 2), 166.7, (C-3), 138.2, 137.3, 133.2, 129.7, 126.4, 126.2, 124.1, 123.6, 123.2, 120.0 (naphthalene C), $52.4\left(\mathrm{OCH}_{2} \mathrm{CH}_{3}\right)$; 34.7 (C-6), 36.8, $\left(\mathrm{CH}_{3}\right), 32.1,24.5(\mathrm{C}-7, \mathrm{C}-5), 24.4\left(2 \mathrm{CH}_{3}\right)$, 16.2 $\left(\mathrm{OCH}_{2} \mathrm{CH}_{3}\right)$; EIMS: $m / z 435[\mathrm{M}]^{+}(46 \%)$; Anal. Calcd for $\mathrm{C}_{24} \mathrm{H}_{25} \mathrm{~N}_{3} \mathrm{O}_{3} \mathrm{~S}$ (435.54): C, 66.18; H, 5.79; N, 9.65; S, 7.36\%. Found: C, 66.84; H, 5.50; N, 9.19; S, 7.47\%.

\section{2. 10. 3. 2-Amino-4-(2-(4-chloronaphthalen-1-yl) hydrazono)-7,7-dimethyl-5-oxo-4,5,6,7-tetra- hydrobenzo $[b]$ thiophene-3-carbonitrile (12c) \\ Pale yellow crystals from ethanol; m.p. $136-139^{\circ} \mathrm{C}$; yield $78 \%$. IR (KBr) cm ${ }^{-1}: 3469-3332\left(\mathrm{NH}, \mathrm{NH}_{2}\right), 3055$, 2985, 2220, 1689, 1663, 1630. ${ }^{1} \mathrm{H}$ NMR $(300 \mathrm{MHz}, \mathrm{DM}-$ SO- $\left.d_{6}\right) \delta 8.39\left(\mathrm{~s}, 1 \mathrm{H}, \mathrm{D}_{2} \mathrm{O}\right.$ exchangeable, $\left.\mathrm{NH}\right), 7.51-7.23(\mathrm{~m}$,}

Mohareb et al.: Synthesis of Heterocyclic Compounds Derived ... 
$6 \mathrm{H}$, naphthalene $\mathrm{H}), 4.88\left(\mathrm{~s}, 2 \mathrm{H}, \mathrm{D}_{2} \mathrm{O}\right.$ exchangeable, $\mathrm{NH}_{2}$ ), $2.40\left(\mathrm{~s}, 2 \mathrm{H}, \mathrm{CH}_{2}\right), 1.09,1.07\left(2 \mathrm{~s}, 6 \mathrm{H}, 2 \mathrm{CH}_{3}\right) ;{ }^{13} \mathrm{C} \mathrm{NMR}$ (DMSO- $\left.d_{6}, 75 \mathrm{MHz}\right) \delta 175.7$ (C-2), 166.2, (C-3), 138.8, 136.6, $133.8,129.3,126.2,126.4,124.2,123.3,122.8,120.2$ (naphthalene $\mathrm{C}), 116.8(\mathrm{CN}), 34.6(\mathrm{C}-6), 32.3\left(\mathrm{CH}_{3}\right), 32.6,24.1$ (C-7, C-5), $24.8\left(2 \mathrm{CH}_{3}\right)$; EIMS: $m / z 408[\mathrm{M}]^{+}(40 \%)$; Anal. Calcd for $\mathrm{C}_{21} \mathrm{H}_{17} \mathrm{ClN}_{4} \mathrm{OS}$ (408.90): C, 61.68; H, 4.19; N, 13.70; S, 7.84\%. Found: C, 61.93; H, 4.26; N, 13.82; S, 7.94\%.

\section{2. 10. 4. Ethyl 2-Amino-4-(2-(4-chloronaphthalen-1- yl)hydrazono)-7,7-dimethyl-5-oxo-4,5,6,7- tetrahydrobenzo $[b]$ thiophene-3-carboxylate (12d)}

Pale brown crystals from acetic acid; m.p. 205-207 ${ }^{\circ} \mathrm{C}$; yield $68 \%$. IR $(\mathrm{KBr}) \mathrm{cm}^{-1}$ : 3442-3329 $\left(\mathrm{NH}, \mathrm{NH}_{2}\right)$, $3055,2985,1689,1687,1663,1630 .{ }^{1} \mathrm{H}$ NMR $(300 \mathrm{MHz}$, DMSO- $\left.d_{6}\right) \delta 8.35\left(\mathrm{~s}, 1 \mathrm{H}, \mathrm{D}_{2} \mathrm{O}\right.$ exchangeable, $\left.\mathrm{NH}\right), 7.53-$ $7.21(\mathrm{~m}, 6 \mathrm{H}$, naphthalene $\mathrm{H}), 4.84\left(\mathrm{~s}, 2 \mathrm{H}, \mathrm{D}_{2} \mathrm{O}\right.$ exchangeable, $\mathrm{NH}_{2}$ ), $4.22\left(\mathrm{q}, 2 \mathrm{H}, J=7.41 \mathrm{~Hz}, \mathrm{OCH}_{2} \mathrm{CH}_{3}\right), 2.41$ (s, $\left.2 \mathrm{H}, \mathrm{CH}_{2}\right), 1.13\left(\mathrm{t}, 3 \mathrm{H}, J=7.41 \mathrm{~Hz}, \mathrm{OCH}_{2} \mathrm{CH}_{3}\right), 1.08,1.05$ $\left(2 \mathrm{~s}, 6 \mathrm{H}, 2 \mathrm{CH}_{3}\right.$ ); ${ }^{13} \mathrm{C}$ NMR (DMSO- $\left.d_{6}, 75 \mathrm{MHz}\right) \delta 175.3$ (C-2), 166.5 (C-3), 138.6, 137.6, 133.5, 129.3, 126.1, 126.0, $124.5, \quad 123.2, \quad 122.7, \quad 120.3$ (naphthalene $\mathrm{C}$ ), 52.5 $\left(\mathrm{OCH}_{2} \mathrm{CH}_{3}\right), 34.8(\mathrm{C}-6), 32.4,24.2(\mathrm{C}-7, \mathrm{C}-5), 24.4\left(2 \mathrm{CH}_{3}\right)$, $16.3\left(\mathrm{OCH}_{2} \mathrm{CH}_{3}\right)$; EIMS: $\mathrm{m} / z 455[\mathrm{M}]^{+}(22 \%)$; Anal. Calcd for $\mathrm{C}_{23} \mathrm{H}_{22} \mathrm{ClN}_{3} \mathrm{O}_{3} \mathrm{~S}$ (455.96): C, 60.59; $\mathrm{H}, 4.86 ; \mathrm{N}, 9.22 ; \mathrm{S}$, 7.03\%. Found: C, 60.52; H, 5.08; N, 9.13; S, 7.24\%.

\section{2. 11. General Procedure for the Synthesis of the Cyclohexane-(1,2,3-triylidene)tris- (hydrazine) Derivatives 13a-d}

To a solution of either 11a $(3.08 \mathrm{~g}, 0.01 \mathrm{~mol}), \mathbf{1 1 b}$ $(3.28 \mathrm{~g}, 0.01 \mathrm{~mol})$ in ethanol $(40 \mathrm{~mL})$ either of hydrazine hydrate $(1.0 \mathrm{~mL}, 0.02 \mathrm{~mol})$ or phenylhydrazine $(3.60 \mathrm{~g}$, $0.02 \mathrm{~mol}$ ) was added. The reaction mixture in each case was heated under reflux for $3 \mathrm{~h}$ then poured onto ice/water containing a few drops of hydrochloric acid and the formed solid product was collected by filtration.

\section{2. 11. 1. (5,5-Dimethyl-2-(2-(4-methylnaphthalen- 1-yl)hydrazono)cyclohexane-1,3-diylidene) bis-(hydrazine) (13a)}

Pale yellow crystals from ethanol; m.p. $220-224^{\circ} \mathrm{C}$; yield $60 \%$. IR $(\mathrm{KBr}) \mathrm{cm}^{-1}: 3473-3326\left(\mathrm{NH}, \mathrm{NH}_{2}\right), 3055$, $2985,1665,1660,1630 .{ }^{1} \mathrm{H}$ NMR $\left(300 \mathrm{MHz}, \mathrm{DMSO}-d_{6}\right) \delta$ $8.26\left(\mathrm{~s}, 1 \mathrm{H}, \mathrm{D}_{2} \mathrm{O}\right.$ exchangeable, $\left.\mathrm{NH}\right), 7.54-7.26(\mathrm{~m}, 6 \mathrm{H}$, naphthalene $\mathrm{H}), 4.51,5.16\left(2 \mathrm{~s}, 4 \mathrm{H}, \mathrm{D}_{2} \mathrm{O}\right.$ exchangeable, $\left.2 \mathrm{NH}_{2}\right), 2.80\left(\mathrm{~s}, 3 \mathrm{H}, \mathrm{CH}_{3}\right), 2.52,2.43\left(2 \mathrm{~s}, 4 \mathrm{H}, 2 \mathrm{CH}_{2}\right), 1.08$, $1.07\left(2 \mathrm{~s}, 6 \mathrm{H}, 2 \mathrm{CH}_{3}\right) ;{ }^{13} \mathrm{C}$ NMR (DMSO- $\left.d_{6}, 75 \mathrm{MHz}\right) \delta$ 176.1, 172.5, 170.3 (C-1, C-2, C-3), 138.3, 137.2, 133.1, 128.5, 126.6, 126.1, 124.8, 123.1, 122.2, 120.3 (naphthalene C), $50.8\left(\mathrm{CH}_{3}\right), 34.3(\mathrm{C}-6), 32.6\left(\mathrm{CH}_{3}\right), 32.8,24.3(\mathrm{C}-7$, C-5), $24.6\left(2 \mathrm{CH}_{3}\right)$; EIMS: $m / z 336[\mathrm{M}]^{+}(35 \%)$; Anal. Calcd for $\mathrm{C}_{19} \mathrm{H}_{24} \mathrm{~N}_{6}$ (336.43): C, 67.83; H, 7.19; N, 24.98\%. Found: C, 67.61; H, 6.93; N, 25.25\%.
2. 2. 11. 2. (2-(2-(4-Chloronaphthalen-1-yl)hydrazono)5,5-dimethylcyclohexane-1,3-diylidene) bis(hydrazine) (13b)

Pale yellow crystals from ethanol; m.p. $210-212{ }^{\circ} \mathrm{C}$; yield $63 \%$. IR $(\mathrm{KBr}) \mathrm{cm}^{-1}: 3486-3348\left(\mathrm{NH}, \mathrm{NH}_{2}\right), 3056$, 2985, 1687, 1665, 1660, 1630. ${ }^{1} \mathrm{H}$ NMR $(300 \mathrm{MHz}, \mathrm{DM}-$ SO- $\left.d_{6}\right) \delta 8.23\left(\mathrm{~s}, 1 \mathrm{H}, \mathrm{D}_{2} \mathrm{O}\right.$ exchangeable, $\left.\mathrm{NH}\right), 7.47-7.22$ (m, 6H, naphthalene $\mathrm{H}), 4.53,5.18\left(2 \mathrm{~s}, 4 \mathrm{H}, \mathrm{D}_{2} \mathrm{O}\right.$ exchangeable, $\left.2 \mathrm{NH}_{2}\right), 2.52,2.43\left(2 \mathrm{~s}, 4 \mathrm{H}, 2 \mathrm{CH}_{2}\right), 1.08,1.07(2 \mathrm{~s}, 6 \mathrm{H}$, $2 \mathrm{CH}_{3}$ ); ${ }^{13} \mathrm{C}$ NMR (DMSO- $\left.d_{6}, 75 \mathrm{MHz}\right) \delta 176.1,172.5$, 170.3 (C-1, C-2, C-3), 138.3, 137.2, 133.1, 128.5, 126.6, $126.1,124.8,123.1,122.2,120.3$ (naphthalene C), 34.6 (C-6), 32.8, 24.5 (C-7, C-5), $24.8\left(2 \mathrm{CH}_{3}\right)$; EIMS: $m / z 356$ $[\mathrm{M}]^{+}(28 \%)$; Anal. Calcd for $\mathrm{C}_{18} \mathrm{H}_{21} \mathrm{ClN}_{6}$ (356.85): C, 60.58; H, 5.93; N, 23.55\%. Found: C, 60.74; H, 6.15; N, 23.68\%.

\section{2. 11. 3. 2,2'-(5,5-Dimethyl-2-(2-(4-} methylnaphthalen-1-yl)hydrazono)cyclohexane-1,3-diylidene)bis(1phenylhydrazine) (13c)

Pale brown crystals from 1,4-dioxan; m.p. 205-207 ${ }^{\circ} \mathrm{C}$; yield $68 \%$. IR $(\mathrm{KBr}) \mathrm{cm}^{-1}$ : $3492-3348\left(\mathrm{NH}, \mathrm{NH}_{2}\right)$, 3055, 2986, 1666, 1660, 1630. ${ }^{1} \mathrm{H}$ NMR $(300 \mathrm{MHz}, \mathrm{DM}-$ SO- $\left.d_{6}\right) \delta 8.36,8.28,8.25\left(3 \mathrm{~s}, 3 \mathrm{H}, \mathrm{D}_{2} \mathrm{O}\right.$ exchangeable, $\left.3 \mathrm{NH}\right)$, 7.56-7.23 (m, $16 \mathrm{H}, 2 \mathrm{C}_{6} \mathrm{H}_{5}$, naphthalene $\left.\mathrm{H}\right), 2.83(\mathrm{~s}, 3 \mathrm{H}$, $\left.\mathrm{CH}_{3}\right), 2.56,2.41\left(2 \mathrm{~s}, 4 \mathrm{H}, 2 \mathrm{CH}_{2}\right), 1.09,1.06\left(2 \mathrm{~s}, 6 \mathrm{H}, 2 \mathrm{CH}_{3}\right)$; ${ }^{13} \mathrm{C}$ NMR (DMSO- $\left.d_{6}, 75 \mathrm{MHz}\right) \delta 176.6,172.8,170.4(\mathrm{C}-1$, C-2, C-3), 138.6, 137.1, 133.0, 128.6, 126.4, 126.1, 125.3, $125.1,124.8,124.6,123.6,123.1,122.6,122.2,121.5,121.2$, $120.3,119.5\left(2 \mathrm{C}_{6} \mathrm{H}_{5}\right.$, naphthalene $\left.\mathrm{C}\right), 50.4\left(\mathrm{CH}_{3}\right), 34.6$ (C-6), 32.6, 24.5 (C-7, C-5), $24.7\left(2 \mathrm{CH}_{3}\right)$; EIMS: $m / z 488$ $[\mathrm{M}]^{+}(40 \%)$; Anal. Calcd for $\mathrm{C}_{31} \mathrm{H}_{32} \mathrm{~N}_{6}$ (488.63): C, 76.20; H, 6.60; N, 17.20\%. Found: C, 76.36; H, 6.39; N, 17.48\%.

\section{2. 11. 4. 2,2'-(2-(2-(4-Chloronaphthalen-1-yl) hydrazono)-5,5-dimethyl-cyclohexane-1,3- diylidene)bis(1-phenylhydrazine) (13d)}

Pale brown crystals from 1,4-dioxan; m.p. 188-191 ${ }^{\circ} \mathrm{C}$; yield $68 \%$. IR $(\mathrm{KBr}) \mathrm{cm}^{-1}$ : 3472-3326 $\left(\mathrm{NH}, \mathrm{NH}_{2}\right)$, 3055, 2985, 1664, 1660, 1630. ${ }^{1} \mathrm{H}$ NMR $(300 \mathrm{MHz}, \mathrm{DM}-$ SO- $\left.d_{6}\right) \delta 8.38,8.23,8.22\left(3 \mathrm{~s}, 3 \mathrm{H}, \mathrm{D}_{2} \mathrm{O}\right.$ exchangeable, $\left.3 \mathrm{NH}\right)$, 7.58-7.24 (m, 16H, 2 $\mathrm{C}_{6} \mathrm{H}_{5}$, naphthalene $\left.\mathrm{H}\right), 2.54,2.46(2 \mathrm{~s}$, $\left.4 \mathrm{H}, 2 \mathrm{CH}_{2}\right), 1.09,1.03\left(2 \mathrm{~s}, 6 \mathrm{H}, 2 \mathrm{CH}_{3}\right) ;{ }^{13} \mathrm{C}$ NMR (DMSO- $\left.d_{6}, 75 \mathrm{MHz}\right) \delta 176.8,172.5,170.3$ (C-1, C-2, C-3), $138.8,137.7,133.1,128.2,126.9,126.3,125.8,125.1,124.3$, $124.6,123.9,123.3,122.6,122.2,122.0,121.8,120.3,119.5$ $\left(2 \mathrm{C}_{6} \mathrm{H}_{5}\right.$, naphthalene $\left.\mathrm{C}\right), 34.8$ (C-6), 32.3, 24.7 (C-7, C-5), $24.5\left(2 \mathrm{CH}_{3}\right)$; EIMS: $m / z 509[\mathrm{M}]^{+}(26 \%)$; Anal. Calcd for $\mathrm{C}_{30} \mathrm{H}_{29} \mathrm{ClN}_{6}$ (509.04): C, 70.78; H, 5.74; N, 16.51\%. Found: C, 70.64; H, 5.59; N, $16.72 \%$.

\section{2. 12. General Procedure for the Synthesis of the Cyclohexane-1,3-dione Dioxime 14a,b}

To a solution of either $11 \mathrm{a}(3.08 \mathrm{~g}, 0.01 \mathrm{~mol}), 11 \mathrm{~b}$ $(3.28 \mathrm{~g}, 0.01 \mathrm{~mol})$ in ethanol $(40 \mathrm{~mL})$ containing sodium 
acetate $(2.0 \mathrm{~g})$ hydroxylamine hydrochloride $(1.440 \mathrm{~g}, 0.02$ mol) was added. The reaction mixture in each case was heated under reflux for $4 \mathrm{~h}$ then poured onto ice/water and the formed solid product was collected by filtration.

\section{2. 12. 1. 5,5-Dimethyl-2-(2-(4-methylnaphthalen- 1-yl)hydrazono)cyclohexane-1,3-dione Dioxime (14a)}

Pale yellow crystals from 1,4-dioxan; m.p. 212-214 ${ }^{\circ} \mathrm{C}$; yield 78\%. IR $(\mathrm{KBr}) \mathrm{cm}^{-1}$ : 3563-3347 (OH, NH), 3055, $2985,1663,1660,1630 .{ }^{1} \mathrm{H}$ NMR $\left(300 \mathrm{MHz}, \mathrm{DMSO}-d_{6}\right) \delta$ 9.68, $10.04\left(2 \mathrm{~s}, 2 \mathrm{H}, \mathrm{D}_{2} \mathrm{O}\right.$ exchangeable, $\left.2 \mathrm{OH}\right), 8.28(\mathrm{~s}, 1 \mathrm{H}$, $\mathrm{D}_{2} \mathrm{O}$ exchangeable, $\left.\mathrm{NH}\right), 7.53-7.28(\mathrm{~m}, 6 \mathrm{H}$, naphthalene $\mathrm{H}), 2.83\left(\mathrm{~s}, 3 \mathrm{H}, \mathrm{CH}_{3}\right), 2.56,2.40\left(2 \mathrm{~s}, 4 \mathrm{H}, 2 \mathrm{CH}_{2}\right), 1.09,1.07$ $\left(2 \mathrm{~s}, 6 \mathrm{H}, 2 \mathrm{CH}_{3}\right) ;{ }^{13} \mathrm{C}$ NMR (DMSO- $\left.d_{6}, 75 \mathrm{MHz}\right) \delta 176.8$, 173.3, 171.8 (C-1, C-2, C-3), 138.6, 137.4, 133.8, 128.2, 126.1, 125.7, 124.8, 123.6, 122.4, 120.1 (naphthalene C), $34.2(\mathrm{C}-6), 32.6\left(\mathrm{CH}_{3}\right), 32.5,24.1(\mathrm{C}-7, \mathrm{C}-5), 24.8\left(2 \mathrm{CH}_{3}\right)$; EIMS: $m / z 338[\mathrm{M}]^{+}(26 \%)$; Anal. Calcd for $\mathrm{C}_{19} \mathrm{H}_{22} \mathrm{~N}_{4} \mathrm{O}_{2}$ (338.40): C, 67.44; H, 6.55; N, 16.56\%. Found: C, 67.64; H, $6.41 ; \mathrm{N}, 16.73 \%$.

\section{2. 12. 2. 2-(2-(4-Chloronaphthalen-1-yl)hydrazono)- 5,5-dimethylcyclohexane-1,3-dione Dioxime (14b)}

Pale yellow crystals from 1,4-dioxan; m.p. 190-193 ${ }^{\circ} \mathrm{C}$; yield 70\%. IR $(\mathrm{KBr}) \mathrm{cm}^{-1}$ : 3533-3329 (OH, NH), 3055, $2985,1665,1662,1630 .{ }^{1} \mathrm{H}$ NMR $\left(300 \mathrm{MHz}\right.$, DMSO- $\left.d_{6}\right) \delta$ 10.06, $9.65\left(2 \mathrm{~s}, 2 \mathrm{H}, \mathrm{D}_{2} \mathrm{O}\right.$ exchangeable, $\left.2 \mathrm{OH}\right), 8.25(\mathrm{~s}, 1 \mathrm{H}$, $\mathrm{D}_{2} \mathrm{O}$ exchangeable, $\left.\mathrm{NH}\right), 7.50-7.24(\mathrm{~m}, 6 \mathrm{H}$, naphthalene $\mathrm{H}), 2.58,2.43\left(2 \mathrm{~s}, 4 \mathrm{H}, 2 \mathrm{CH}_{2}\right), 1.09,1.06\left(2 \mathrm{~s}, 6 \mathrm{H}, 2 \mathrm{CH}_{3}\right)$; ${ }^{13} \mathrm{C}$ NMR (DMSO- $\left.d_{6}, 75 \mathrm{MHz}\right) \delta 176.5,173.6,171.8(\mathrm{C}-1$, C-2, C-3), 138.8, 137.1, 133.5, 128.0, 126.7, 125.3, 124.8, 123.4, 122.6, 120.1 (naphthalene C), 34.5 (C-6), 32.5, 24.3 (C-7, C-5), $24.5\left(2 \mathrm{CH}_{3}\right)$; EIMS: $m / z 358[\mathrm{M}]^{+}(32 \%)$; Anal. Calcd for $\mathrm{C}_{18} \mathrm{H}_{19} \mathrm{ClN}_{4} \mathrm{O}_{2}$ (358.82): C, 60.25; H, 5.34; N, $15.61 \%$. Found: C, 60.19; H, 5.28; N, 15.80\%.

\section{2. 13. General Procedure for the Synthesis of the 2,3,5,6,7,8-Hexahydrocinnoline Derivatives 16a-d}

To a solution of either of $11 \mathrm{a}(3.08 \mathrm{~g}, 0.01 \mathrm{~mol}), \mathbf{1 1 b}$ $(3.28 \mathrm{~g}, 0.01 \mathrm{~mol})$ in ethanol $(40 \mathrm{~mL})$ containing triethylamine either malononitrile $(0.66 \mathrm{~g}, 0.01 \mathrm{~mol})$ or ethyl cyanoacetate $(1.07 \mathrm{~g}, 0.01 \mathrm{~mol})$ was added. The reaction mixture in each case was heated under reflux for $3 \mathrm{~h}$ then poured onto ice/water containing a few drops of hydrochloric acid and the formed solid product was collected by filtration.

\section{2. 13. 1. 3-Imino-6,6-dimethyl-2-(4- methylnaphthalen-1-yl)-8-oxo-2,3,5,6,7,8- hexahydrocinnoline-4-carbonitrile (16a)}

Pale brown crystals from 1,4-dioxan; m.p. 188-191 ${ }^{\circ} \mathrm{C}$; yield $70 \%$. IR $(\mathrm{KBr}) \mathrm{cm}^{-1}$ : 3478-3337 (NH), 3055,
$2985,2220,1687,1666,1660,1630 .{ }^{1} \mathrm{H}$ NMR $(300 \mathrm{MHz}$, DMSO- $\left.d_{6}\right) \delta 8.25\left(\mathrm{~s}, 1 \mathrm{H}, \mathrm{D}_{2} \mathrm{O}\right.$ exchangeable, $\left.\mathrm{NH}\right), 7.53-$ $7.24(\mathrm{~m}, 6 \mathrm{H}$, naphthalene $\mathrm{H}), 2.86\left(\mathrm{~s}, 3 \mathrm{H}, \mathrm{CH}_{3}\right), 2.53,2.42$ $\left(2 \mathrm{~s}, 4 \mathrm{H}, 2 \mathrm{CH}_{2}\right), 1.09,1.07\left(2 \mathrm{~s}, 6 \mathrm{H}, 2 \mathrm{CH}_{3}\right) ;{ }^{13} \mathrm{C} \mathrm{NMR}$ (DMSO- $\left.d_{6}, 75 \mathrm{MHz}\right) \delta 176.4$ (C-3), 165.8 (C-8), 138.9, $136.3,135.4,128.1,126.5,125.3,124.8,123.6,122.4,120.4$ (naphthalene C), $117.0(\mathrm{CN}), 34.6(\mathrm{C}-6), 32.8\left(\mathrm{CH}_{3}\right), 32.2$, 24.3 (C-7, C-5), $24.8\left(2 \mathrm{CH}_{3}\right)$; EIMS: $m / z 356[\mathrm{M}]^{+}(32 \%)$; Anal. Calcd for $\mathrm{C}_{22} \mathrm{H}_{20} \mathrm{~N}_{4} \mathrm{O}$ (356.42): C, 74.14; $\mathrm{H}, 5.66 ; \mathrm{N}$, 15.72\%. Found: C, 74.03; H, 5.79; N, 15.84\%.

\section{2. 13. 2. 6,6-Dimethyl-2-(4-methylnaphthalen-1-yl)-} 3,8-dioxo-2,3,5,6,7,8-hexahydrocinnoline-4carbonitrile (16b)

Pale yellow crystals from 1,4-dioxan; m.p. 203-206 ${ }^{\circ} \mathrm{C}$; yield 65\%. IR (KBr) cm ${ }^{-1}: 3055,2985,2220,1689,1666$, $1660,1630 .{ }^{1} \mathrm{H}$ NMR $\left(300 \mathrm{MHz}, \mathrm{DMSO}-d_{6}\right) \delta 7.56-7.23$ $(\mathrm{m}, 6 \mathrm{H}$, naphthalene $\mathrm{H}), 2.84\left(\mathrm{~s}, 3 \mathrm{H}, \mathrm{CH}_{3}\right), 2.56,2.40(2 \mathrm{~s}$, $\left.4 \mathrm{H}, 2 \mathrm{CH}_{2}\right), \quad 1.09,1.08\left(2 \mathrm{~s}, 6 \mathrm{H}, 2 \mathrm{CH}_{3}\right) ;{ }^{13} \mathrm{C} \mathrm{NMR}$ $\left(\mathrm{DMSO}-d_{6}, 75 \mathrm{MHz}\right) \delta 176.6$ (C-3), 165.5 (C-8), 138.4, $137.1,135.2,127.4,126.5,125.1,124.8,123.6,122.2,120.4$ (naphthalene C), $116.8(\mathrm{CN}), 34.6(\mathrm{C}-6), 32.5\left(\mathrm{CH}_{3}\right), 32.0$, 24.6 (C-7, C-5), $24.5\left(2 \mathrm{CH}_{3}\right)$; EIMS: $m / z 357[\mathrm{M}]^{+}(28 \%)$; Anal. Calcd for $\mathrm{C}_{22} \mathrm{H}_{19} \mathrm{~N}_{3} \mathrm{O}_{2}(357.41)$ : C, 73.93; H, 5.36; N, $11.76 \%$. Found: C, 73.61; H, 5.49; N, 11.83\%.

\section{2. 13. 3. 2-(4-Chloronaphthalen-1-yl)-3- imino-6,6-dimethyl-8-oxo-2,3,5,6,7,8- hexahydrocinnoline-4-carbonitrile (16c)}

Pale yellow crystals from 1,4-dioxan; m.p. 180-183 ${ }^{\circ} \mathrm{C}$; yield $65 \%$. IR $(\mathrm{KBr}) \mathrm{cm}^{-1}$ : 3459-3341 (NH), 3055, 2985, 2220, 1689, 1664, 1660, 1630. ${ }^{1} \mathrm{H}$ NMR $(300 \mathrm{MHz}$, DMSO- $\left.d_{6}\right) \delta 8.23\left(\mathrm{~s}, 1 \mathrm{H}, \mathrm{D}_{2} \mathrm{O}\right.$ exchangeable, $\left.\mathrm{NH}\right), 7.56-$ $7.23(\mathrm{~m}, 6 \mathrm{H}$, naphthalene $\mathrm{H}), 2.56,2.42\left(2 \mathrm{~s}, 4 \mathrm{H}, 2 \mathrm{CH}_{2}\right)$, $1.09,1.07\left(2 \mathrm{~s}, 6 \mathrm{H}, 2 \mathrm{CH}_{3}\right) ;{ }^{13} \mathrm{C}$ NMR (DMSO- $\left.d_{6}, 75 \mathrm{MHz}\right)$ $\delta$ 176.6 (C-3), $165.9(\mathrm{C}-8), 138.5,137.8,135.3,127.6,126.2$, 125.1, 124.8, 123.4, 122.1, 120.5 (naphthalene C), 116.8 (CN), 34.4 (C-6), 32.0, 24.7 (C-7, C-5), $24.5\left(2 \mathrm{CH}_{3}\right)$; EIMS: $\mathrm{m} / z 376[\mathrm{M}]^{+}(32 \%)$; Anal. Calcd for $\mathrm{C}_{21} \mathrm{H}_{17} \mathrm{ClN}_{4} \mathrm{O}$ (376.84): C, 66.93; H, 4.55; N, 14.87\%. Found: C, 67.27; H, $4.74 ; \mathrm{N}, 15.04 \%$.

\section{2. 13. 4. 2-(4-Chloronaphthalen-1-yl)-6,6-dimethyl- 3,8-dioxo-2,3,5,6,7,8-hexahydrocinnoline-4- carbonitrile (16d)}

Pale yellow crystals from 1,4-dioxan; m.p. 210-213 ${ }^{\circ} \mathrm{C}$; yield 70\%. IR (KBr) cm ${ }^{-1}: 3055,2985,2220,1689,1686$, $1663,1660,1630 .{ }^{1} \mathrm{H}$ NMR $\left(200 \mathrm{MHz}, \mathrm{DMSO}-d_{6}\right) \delta 7.58-$ $7.21(\mathrm{~m}, 6 \mathrm{H}$, naphthalene $\mathrm{H}), 2.54,2.43\left(2 \mathrm{~s}, 4 \mathrm{H}, 2 \mathrm{CH}_{2}\right)$, $1.09,1.08\left(2 \mathrm{~s}, 6 \mathrm{H}, 2 \mathrm{CH}_{3}\right) ;{ }^{13} \mathrm{C}$ NMR (DMSO- $\left.d_{6}, 75 \mathrm{MHz}\right)$ $\delta 176.8(\mathrm{C}-3), 165.3(\mathrm{C}-8), 138.6,137.5,135.2,127.8,126.2$, 125.6, 124.3, 123.4, 122.1, 120.1 (naphthalene C), 116.8 (CN), 34.4 (C-6), 32.2, 24.3 (C-7, C-5), $24.5\left(2 \mathrm{CH}_{3}\right)$; EIMS: $\mathrm{m} / z 377[\mathrm{M}]^{+}(30 \%)$; Anal. Calcd for $\mathrm{C}_{21} \mathrm{H}_{16} \mathrm{ClN}_{3} \mathrm{O}_{2}$ (377.82): C, 66.76; H, 4.27; N, 11.12\%. Found: C, 66.92; H, $4.58 ; \mathrm{N}, 11.43 \%$. 


\section{3. Biology Section}

\section{3. 1. In vitro Cell Assays}

All the synthesized compounds were assessed for the inhibitory activities against A549 (non-small cell lung cancer), H460 (human lung cancer), HT-29 (human colon cancer) and MKN-45 (human gastric cancer) cancer cell lines together with foretinib as the positive control by a MTT assay. Furthermore, all compounds were further evaluated against U87MG (human glioblastoma) and SMMC-7721 (human liver cancer) cell lines. The results expressed as $\mathrm{IC}_{50}$ are summarized in Table 2 . The $\mathrm{IC}_{50}$ values are the average of at least three independent experiments. The data listed in Table 2 reveal that the compounds possess moderate to strong cytotoxicity against the five tested cell lines in the single-digit nM range, and high selectivity for inhibition against A549, H460 and MKN-45 cells. The promising compounds were $3 \mathbf{c}, 5 \mathbf{b}, 5 \mathbf{e}, \mathbf{5 f}, \mathbf{7 c}, \mathbf{7 f}$, 9c, 11b, 12c, 12d, 13b, 13d, 14b, 16c and 16d among the tested compounds.

\section{3. 1. 1. Structure Activity Relationship}

From Table 1 it is clear that most of compounds showed high cytotoxicities against the six cancer cell lines. In most cases the high inhibitions was due to the presence of electronegative substituent through the aryl or the heterocyclic rings. Considering the benzylidine derivatives $\mathbf{3 a}-\mathbf{c}$, it is obvious that compound $\mathbf{3 a}(\mathrm{X}=\mathrm{H})$ is of low inhibitions relative to $\mathbf{3 b}\left(\mathrm{X}=\mathrm{OCH}_{3}\right)$ and $\mathbf{3} \mathbf{c}(\mathrm{X}=\mathrm{Cl})$. In addition, compound $\mathbf{3 b}$ showed moderate inhibitions and 3a with highest inhibitions among the three compounds. Considering the thiophene derivatives $\mathbf{5 a} \mathbf{a} \mathbf{f}$, it is clear that compounds 5d $\left(\mathrm{X}=\mathrm{OCH}_{3}, \mathrm{R}=\mathrm{COOEt}\right), \mathbf{5 e}(\mathrm{X}=\mathrm{Cl}, \mathrm{R}=$ $\mathrm{CN})$ and $\mathbf{5 f}(\mathrm{X}=\mathrm{Cl}, \mathrm{R}=\mathrm{COOEt})$ were the most cytotoxic compounds. On the other hand, for the 4,5,6,7-tetrahydro- $2 \mathrm{H}$-indazole derivatives $\mathbf{7 a - f}$, where compounds $7 \mathbf{c}$

Table 1. In vitro growth inhibitory effects $\mathrm{IC}_{50} \pm \operatorname{SEM}(\mu \mathrm{M})$ of the newly synthesized compounds towards cancer cell lines

\begin{tabular}{|c|c|c|c|c|c|c|}
\hline \multirow[t]{2}{*}{ Compound No } & \multicolumn{6}{|c|}{$\mathrm{IC}_{50} \pm \mathrm{SEM}(\mu \mathrm{M})$} \\
\hline & A549 & H460 & HT29 & MKN-45 & U87MG & SMMC-7721 \\
\hline $3 \mathbf{a}$ & $8.32 \pm 2.57$ & $8.68 \pm 2.60$ & $7.93 \pm 2.49$ & $8.73 \pm 2.71$ & $8.48 \pm 2.90$ & $8.29 \pm 2.09$ \\
\hline $3 \mathbf{b}$ & $2.63 \pm 1.14$ & $2.64 \pm 0.95$ & $1.63 \pm 0.59$ & $1.47 \pm 0.69$ & $2.16 \pm 0.96$ & $1.53 \pm 0.87$ \\
\hline $3 c$ & $0.29 \pm 0.12$ & $0.34 \pm 0.18$ & $0.62 \pm 0.36$ & $0.26 \pm 0.19$ & $0.35 \pm 0.22$ & $0.41 \pm 0.13$ \\
\hline $5 \mathbf{a}$ & $5.23 \pm 1.47$ & $4.82 \pm 1.36$ & $2.58 \pm 0.926$ & $3.62 \pm 1.21$ & $5.78 \pm 1.30$ & $5.39 \pm 1.15$ \\
\hline $5 \mathbf{b}$ & $6.88 \pm 1.23$ & $4.83 \pm 2.05$ & $5.64 \pm 2.11$ & $4.52 \pm 2.18$ & $2.38 \pm 2.21$ & $4.20 \pm 1.19$ \\
\hline $5 c$ & $4.56 \pm 1.25$ & $6.72 \pm 1.81$ & $6.23 \pm 2.32$ & $5.88 \pm 1.41$ & $5.22 \pm 2.30$ & $2.34 \pm 1.29$ \\
\hline $5 d$ & $1.20 \pm 0.74$ & $1.47 \pm 0.47$ & $1.23 \pm 0.63$ & $2.22 \pm 1.15$ & $1.60 \pm 0.86$ & $2.96 \pm 1.63$ \\
\hline $5 e$ & $0.41 \pm 0.22$ & $0.87 \pm 0.53$ & $0.52 \pm 0.25$ & $0.43 \pm 0.21$ & $0.39 \pm 0.16$ & $0.48 \pm 0.25$ \\
\hline $5 f$ & $0.26 \pm 0.17$ & $0.31 \pm 0.15$ & $0.42 \pm 0.32$ & $0.26 \pm 0.15$ & $0.40 \pm 0.26$ & $0.38 \pm 0.15$ \\
\hline $7 \mathbf{a}$ & $8.90 \pm 3.60$ & $9.53 \pm 2.06$ & $8.31 \pm 2.70$ & $6.16 \pm 1.93$ & $6.49 \pm 2.52$ & $8.24 \pm 3.19$ \\
\hline $\mathbf{7 b}$ & $2.36 \pm 1.06$ & $1.48 \pm 0.69$ & $2.40 \pm 1.13$ & $1.05 \pm 0.72$ & $1.28 \pm 0.69$ & $2.59 \pm 1.06$ \\
\hline $7 \mathrm{c}$ & $0.43 \pm 0.24$ & $0.57 \pm 0.16$ & $0.42 \pm 0.19$ & $0.58 \pm 0.21$ & $0.35 \pm 0.22$ & $0.28 \pm 0.09$ \\
\hline $7 d$ & $3.25 \pm 1.19$ & $2.36 \pm 0.92$ & $3.50 \pm 1.56$ & $2.87 \pm 1.40$ & $3.42 \pm 1.82$ & $1.92 \pm 0.89$ \\
\hline $7 e$ & $1.28 \pm 0.42$ & $1.67 \pm 0.83$ & $2.61 \pm 0.76$ & $1.88 \pm 0.42$ & $2.26 \pm 0.80$ & $1.53 \pm 0.80$ \\
\hline $7 f$ & $0.24 \pm 0.11$ & $0.31 \pm 0.16$ & $0.38 \pm 0.27$ & $0.41 \pm 0.12$ & $0.25 \pm 0.08$ & $0.52 \pm 0.16$ \\
\hline $9 a$ & $8.38 \pm 2.72$ & $7.29 \pm 2.60$ & $8.53 \pm 2.30$ & $7.93 \pm 2.27$ & $8.09 \pm 1.74$ & $6.92 \pm 1.79$ \\
\hline $9 b$ & $1.18 \pm 0.30$ & $0.86 \pm 0.53$ & $0.69 \pm 0.40$ & $0.83 \pm 0.27$ & $0.59 \pm 0.31$ & $0.63 \pm 0.25$ \\
\hline $9 c$ & $0.32 \pm 0.17$ & $0.52 \pm 0.13$ & $0.44 \pm 0.15$ & $0.23 \pm 0.13$ & $0.23 \pm 0.42$ & $0.63 \pm 0.30$ \\
\hline $11 \mathrm{a}$ & $1.03 \pm 0.36$ & $1.43 \pm 0.39$ & $0.96 \pm 1.42$ & $0.78 \pm 0.35$ & $0.68 \pm 0.34$ & $0.80 \pm 0.35$ \\
\hline $11 b$ & $0.28 \pm 0.19$ & $0.36 \pm 0.18$ & $0.41 \pm 0.27$ & $0.31 \pm 0.13$ & $0.28 \pm 0.15$ & $0.50 \pm 0.21$ \\
\hline $12 a$ & $2.25 \pm 0.68$ & $2.70 \pm 0.61$ & $1.09 \pm 0.79$ & $1.17 \pm 0.40$ & $1.58 \pm 0.54$ & $1.80 \pm 0.93$ \\
\hline $12 b$ & $4.27 \pm 1.12$ & $3.55 \pm 1.25$ & $2.38 \pm 1.16$ & $3.42 \pm 1.38$ & $2.25 \pm 1.68$ & $3.51 \pm 1.09$ \\
\hline $12 \mathrm{c}$ & $0.46 \pm 0.18$ & $0.39 \pm 0.17$ & $0.72 \pm 0.23$ & $0.84 \pm 0.26$ & $0.34 \pm 0.18$ & $0.34 \pm 0.29$ \\
\hline $12 d$ & $0.63 \pm 0.24$ & $0.22 \pm 0.13$ & $0.47 \pm 0.17$ & $0.68 \pm 0.16$ & $0.37 \pm 0.24$ & $0.52 \pm 0.20$ \\
\hline $13 a$ & $6.09 \pm 1.26$ & $7.83 \pm 1.84$ & $8.39 \pm 2.53$ & $6.73 \pm 1.80$ & $8.53 \pm 2.06$ & $5.27 \pm 1.73$ \\
\hline $13 b$ & $0.98 \pm 0.32$ & $0.45 \pm 0.25$ & $0.69 \pm 0.32$ & $0.38 \pm 0.16$ & $0.50 \pm 0.17$ & $0.68 \pm 0.42$ \\
\hline $13 c$ & $4.48 \pm 1.23$ & $5.59 \pm 1.27$ & $3.27 \pm 1.24$ & $4.25 \pm 1.56$ & $2.82 \pm 1.04$ & $3.53 \pm 1.51$ \\
\hline $13 d$ & $0.34 \pm 0.26$ & $0.39 \pm 0.25$ & $0.60 \pm 0.42$ & $0.52 \pm 0.20$ & $0.28 \pm 0.19$ & $0.26 \pm 0.15$ \\
\hline $14 a$ & $6.23 \pm 1.38$ & $5.39 \pm 1.13$ & $5.09 \pm 1.25$ & $4.78 \pm 2.21$ & $5.42 \pm 2.32$ & $6.26 \pm 2.63$ \\
\hline $14 b$ & $0.79 \pm 0.35$ & $0.85 \pm 0.28$ & $0.84 \pm 0.31$ & $0.59 \pm 0.36$ & $0.80 \pm 0.31$ & $0.48 \pm 0.24$ \\
\hline $16 a$ & $7.28 \pm 2.09$ & $8.26 \pm 3.16$ & $9.26 \pm 2.41$ & $6.27 \pm 1.89$ & $8.47 \pm 2.53$ & $6.61 \pm 1.75$ \\
\hline $16 b$ & $4.33 \pm 1.70$ & $4.16 \pm 1.72$ & $2.46 \pm 1.29$ & $4.59 \pm 1.25$ & $3.72 \pm 1.47$ & $4.64 \pm 1.63$ \\
\hline $16 c$ & $0.28 \pm 0.18$ & $0.43 \pm 0.15$ & $0.34 \pm 0.12$ & $0.45 \pm 0.22$ & $0.39 \pm 0.28$ & $0.39 \pm 0.132$ \\
\hline $16 d$ & $0.23 \pm 0.12$ & $0.44 \pm 0.20$ & $0.32 \pm 0.17$ & $0.28 \pm 0.08$ & $0.20 \pm 0.14$ & $0.19 \pm 0.017$ \\
\hline foretinib & $0.08 \pm 0.01$ & $0.18 \pm 0.03$ & $0.15 \pm 0.023$ & $0.03 \pm 0.0055$ & $0.90 \pm 0.13$ & $0.44 \pm 0.062$ \\
\hline
\end{tabular}


$(\mathrm{R}=\mathrm{H}, \mathrm{X}=\mathrm{Cl})$ and $7 \mathbf{f}(\mathrm{R} \mathrm{Ph}, \mathrm{X}=\mathrm{Cl})$ were the most cytotoxic compounds. For the isoxazole derivatives $\mathbf{9 a}-\mathbf{c}, \mathbf{c o m}-$ pound 9c $(\mathrm{X}=\mathrm{Cl})$ was the most cyotoxic compound among the three compounds. While compound $\mathbf{9 b}(\mathrm{X}=$ $\mathrm{OCH}_{3}$ ) has high cytotoxiciy against the five cancer cell lines H460, HT29, MKN-45, U87MG and SMMC-7721 with $\mathrm{IC}_{50}$ values $0.86,0.69,0.83,0.59$ and $0.63 \mu \mathrm{M}$, respectively. For the arylhydrazone derivatives 11a,b, compound 11b with $\mathrm{Y}=\mathrm{Cl}$ was more cytotoxic than 11a with $\mathrm{Y}=$ $\mathrm{CH}_{3}$. Considering the fused thiophene derivatives 12a-d, it is obvious that compounds $\mathbf{1 2 c}(\mathrm{Y}=\mathrm{Cl}, \mathrm{R}=\mathrm{CN})$ and $\mathbf{1 2 d}$ ( $\mathrm{Y}=\mathrm{Cl}, \mathrm{R}=\mathrm{COOEt}$ ) were more cytotoxic than compounds $12 \mathbf{a}$ and $\mathbf{1 2 b}$ although compound 12a has moderate cytotoxicity. In case of the trihydrazone derivatives 13a-d, compounds $13 \mathbf{b}(\mathrm{R}=\mathrm{H}, \mathrm{Y}=\mathrm{Cl})$ and $13 \mathbf{d}(\mathrm{R}=\mathrm{Ph}$, $\mathrm{Y}=\mathrm{Cl}$ ) were the most cytotoxic compounds, it is clear that the presence of $\mathrm{Cl}$ group was responsible for such high cytotoxicity. The same was also observed in the case of $\mathbf{1 4 a}, \mathbf{b}$ where compound $\mathbf{1 4 b}(\mathrm{Y}=\mathrm{Cl})$ was more cytotoxic than 14a $\left(\mathrm{Y}=\mathrm{CH}_{3}\right)$. Finally, for the fused pyridazine derivatives 16a-d, it is clear that compounds $\mathbf{1 6 c}\left(\mathrm{Y}=\mathrm{Cl}, \mathrm{R}^{\mathrm{c}}=\mathrm{NH}\right)$ and $\mathbf{1 6 d}\left(\mathrm{Y}=\mathrm{Cl}, \mathrm{R}^{\prime}=\mathrm{O}\right)$ were the most cytotoxic compounds among the four compounds.

\section{3. 2. Inhibition of Tyrosine Kinases (Enzyme $\left.\mathrm{IC}_{50}(\mathrm{nM})\right)$}

Compounds 3c, 5b, 5e, 5f, 7c, 7f, 9c, 11b, 12c, 12d, 13b, 13d, 14b, 16c and 16d were the most potent compounds against the selected six cancer cell lines and were further tested toward the five tyrosine kinases c-kit, Flt-3, VEGFR-2, EGFR and PDGFR and the data are shown in Table 2.

The selection of the five tyrosine kinases was based on the fact that these contain seven, five and three Ig-like domains in the extracellular domain, respectively. ${ }^{20}$ These RTKs have been implicated in vascular development by affecting the proliferation and migration of endothelial cells or parricides. Among them, VEGFR is a major regulator of tumor angiogenesis via endothelial cell proliferation and the permeability of blood vessels. ${ }^{21,22}$ VEGFR is expressed in most human cancers such as breast, kidney and colon and patients with tumors showing elevated VEGFR expression have a poor prognosis. ${ }^{23}$ It is clear that compounds 5b, 5e, 5f, 7c, 11b, 12c, 12d, 13d, 14b, 16c and $16 \mathbf{d}$ are the most inhibitory compounds.

\section{3. 4. Inhibition of Selected Compounds Towards Pim-1 Kinase}

Furthermore, compounds $5 \mathrm{~b}, 5 \mathrm{e}, \mathbf{5 f}, \mathbf{7 c}, \mathbf{1 1 b}, \mathbf{1 2 c}$, 12d, 13d, 14b, 16c and 16d were selected to examine their Pim-1 kinase inhibition activity (Table 3 ) as these compounds showed high inhibition toward the tested cancer cell lines at a range and high inhibitions toward the five

Table 3. Inhibition of Pim-1 kinase by compounds $5 \mathbf{b}, \mathbf{5 e}, \mathbf{5 f}, \mathbf{7 c}$, $11 b, 12 c, 12 d, 13 d, 14 b, 16 c$ and 16d 5b

\begin{tabular}{ccc}
\hline Compound & $\begin{array}{c}\text { Inhibition ratio } \\
\text { at } \mathbf{1 0} \boldsymbol{\mu M}\end{array}$ & IC $_{\mathbf{5 0}}(\boldsymbol{\mu M})$ \\
\hline $\mathbf{5 b}$ & 96 & 0.31 \\
$\mathbf{5 e}$ & 26 & $>10$ \\
$\mathbf{5 f}$ & 22 & $>10$ \\
$\mathbf{7 c}$ & 28 & $>10$ \\
$\mathbf{1 1 b}$ & 86 & 0.68 \\
$\mathbf{1 2 c}$ & 28 & $>10$ \\
$\mathbf{1 2 d}$ & 92 & 0.42 \\
$\mathbf{1 3 d}$ & 94 & 0.38 \\
$\mathbf{1 4 b}$ & 89 & 0.46 \\
$\mathbf{1 6}$ & 95 & 0.34 \\
$\mathbf{1 6 d}$ & 23 & $>10$ \\
SGI-1776 & - & 0.048 \\
\hline
\end{tabular}

Table 2. Inhibition of tyrosine kinases (Enzyme $\mathrm{IC}_{50}(\mathrm{nM})$ by compounds $\mathbf{3 c}, \mathbf{5 b}, \mathbf{7 c}, \mathbf{7 f}, \mathbf{9 c}, \mathbf{1 1} \mathbf{b}, \mathbf{1 2 c}$, $12 d, 13 b, 13 d, 14 b, 16 c$ and $16 d$

\begin{tabular}{cccccc}
\hline Compound & c-Kit & Flt-3 & VEGFR-2 & EGFR & PDGFR \\
\hline $\mathbf{3 c}$ & 1.61 & 0.96 & 1.36 & 1.42 & 1.17 \\
$\mathbf{5 b}$ & 0.35 & 0.27 & 0.21 & 0.36 & 0.42 \\
$\mathbf{5 e}$ & 0.28 & 0.19 & 0.36 & 0.25 & 0.46 \\
$\mathbf{5 f}$ & 0.37 & 0.24 & 0.42 & 0.39 & 0.48 \\
$\mathbf{7 c}$ & 0.42 & 0.36 & 0.51 & 0.28 & 0.26 \\
$\mathbf{7 f}$ & 1.09 & 1.24 & 1.31 & 0.96 & 0.30 \\
$\mathbf{9 c}$ & 1.16 & 1.27 & 1.40 & 1.16 & 0.52 \\
$\mathbf{1 1 b}$ & 0.28 & 0.50 & 0.19 & 0.33 & 0.26 \\
$\mathbf{1 2 c}$ & 0.26 & 0.38 & 0.51 & 0.39 & 0.27 \\
$\mathbf{1 2 d}$ & 0.30 & 0.35 & 0.19 & 0.43 & 0.60 \\
$\mathbf{1 3 b}$ & 1.01 & 0.82 & 1.07 & 1.53 & 1.32 \\
$\mathbf{1 3 d}$ & 0.38 & 0.26 & 0.63 & 0.62 & 0.47 \\
$\mathbf{1 4 b}$ & 0.47 & 0.56 & 0.32 & 0.53 & 0.61 \\
$\mathbf{1 6 c}$ & 0.24 & 0.26 & 0.38 & 0.41 & 0.37 \\
$\mathbf{1 6 d}$ & 0.72 & 0.69 & 0.93 & 0.52 & 0.73 \\
\hline
\end{tabular}

Mohareb et al.: Synthesis of Heterocyclic Compounds Derived ... 
tyrosine kinases. Compounds 5b, 11b, 12d, 13d, 14b and $16 \mathrm{c}$ were the most potent to inhibit Pim-1 activity with $\mathrm{IC}_{50}$ value of $0.31,0.68,0.42,0.38,0.46$ and $0.34 \mu \mathrm{M}$, while $\mathbf{7 c}, \mathbf{5 e}, \mathbf{5 f}, \mathbf{1 2 c}$ and $\mathbf{1 6 d}$ were less effective $\left(\mathrm{IC}_{50}>10 \mu \mathrm{M}\right)$. SGI-1776 was used as the positive control with $\mathrm{IC}_{50} 0.048$ $\mu \mathrm{M}$ in the assay. These profiles in combination with cell growth inhibition data of the tested compounds are listed in Table 3 indicating that Pim-1 was a potential target of these compounds.

\section{Results and Discussion}

The synthetic route to prepare a new class of biologically active molecules using dimedone as the key starting compound is illustrated in Schemes 1-3. The reaction of dimedone (1) with any of the aromatic aldehydes $\mathbf{2 a - c}$ gave the benzylidene derivatives $\mathbf{3 a}-\mathbf{c}$, respectively. Compounds 3a-c were appropriate for Gewald's thiophene synthe$\mathrm{sis}^{24-26}$ through the reaction of any of compounds $3 \mathbf{a}-\mathbf{c}$ with either of malononitrile (4a) or ethyl cyanoacetate $(\mathbf{4 b})$ and elemental sulfur gave the 6,7-dihydrobenzo[b]thiophen-5(4H)-one derivatives 5a-f, respectively. The structures of compounds $\mathbf{5 a}-\mathbf{f}$ were based on their analytical and spectral data. Thus, the ${ }^{1} \mathrm{H}$ NMR spectrum of compound $\mathbf{5 a}$ (as an example) showed the presence of one $\mathrm{NH}_{2}$ group at $\delta 4.30 \mathrm{ppm}\left(\mathrm{D}_{2} \mathrm{O}\right.$ exchangeable $)$ and a singlet at $\delta$ $2.32 \mathrm{ppm}$ indicating one $\mathrm{CH}_{2}$ group. The ${ }^{13} \mathrm{C}$ NMR spectrum revealed the presence of a signal at 166.0 due to the presence of $\mathrm{C}=\mathrm{O}$ group and signals at $\delta 138.6,136.0,135.2$, $130.7,127.8,125.4,124.3,121.0$ due to the phenyl and thiophene carbons. On the other hand, the reaction of any of<smiles>CC1(C)CC(=O)CC(=O)C1</smiles>

1

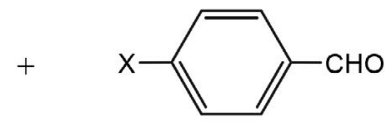

$$
\begin{aligned}
& \text { 2a, } \mathrm{X}=\mathrm{H} \\
& \text { b, } \mathrm{X}=\mathrm{OCH}_{3} \\
& \text { c, } \mathrm{X}=\mathrm{Cl}
\end{aligned}
$$

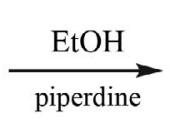<smiles>[X]c1ccc(C=C2C(=O)CC(C)(C)CC2=O)cc1</smiles>

3a, $\mathrm{X}=\mathrm{H}$

b, $\mathrm{X}=\mathrm{OCH}_{3}$

c, $\mathrm{X}=\mathrm{Cl}$<smiles>[X]c1ccc(C=C2C(=O)CC(C)(C)c3sc(N)c([R])c32)cc1</smiles>

$$
\begin{aligned}
\mathbf{4 a}, \mathrm{R} & =\mathrm{CN} \\
\mathbf{b}, \mathrm{R} & =\mathrm{COOEt}
\end{aligned}
$$

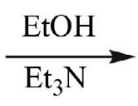

$$
\begin{aligned}
\text { 5a, } \mathrm{X} & =\mathrm{H}, \mathrm{R}=\mathrm{CN} \\
\text { b, } \mathrm{X} & =\mathrm{H}, \mathrm{R}=\mathrm{COOEt} \\
\text { c, } \mathrm{X} & =\mathrm{OCH}_{3}, \mathrm{R}=\mathrm{CN} \\
\text { d, } \mathrm{X} & =\mathrm{OCH}_{3}, \mathrm{R}=\mathrm{COOEt} \\
\text { e, } \mathrm{X} & =\mathrm{Cl}, \mathrm{R}=\mathrm{CN} \\
\text { f, } \mathrm{X} & =\mathrm{Cl}, \mathrm{R}=\mathrm{COOEt}
\end{aligned}
$$

Scheme 1. Synthesis of compounds $3 \mathbf{a}-\mathbf{c}, 5 \mathbf{a}-\mathbf{f}$ and $7 \mathbf{a}-\mathbf{f}$.<smiles>[R]NC1CC(C)(C)Cc2n[nH]c(-c3ccc([X])cc3)c21</smiles>

$$
\begin{aligned}
\text { 7a, } \mathrm{R} & =\mathrm{H}, \mathrm{X}=\mathrm{H} \\
\text { b, } \mathrm{R} & =\mathrm{H}, \mathrm{X}=\mathrm{OCH}_{3} \\
\text { c, } \mathrm{R} & =\mathrm{H}, \mathrm{X}=\mathrm{Cl} \\
\text { d, } \mathrm{R} & =\mathrm{Ph}, \mathrm{X}=\mathrm{H} \\
\text { e, } \mathrm{R} & =\mathrm{Ph}, \mathrm{X}=\mathrm{OCH}_{3} \\
\text { f, } \mathrm{R} & =\mathrm{Ph}, \mathrm{X}=\mathrm{Cl}
\end{aligned}
$$


compounds $\mathbf{3 a}-\mathbf{c}$ with either of hydrazine hydrate $(\mathbf{6 a})$ or phenylhydrazine (6b) gave the 4,5,6,7-tetrahydro- $2 \mathrm{H}$-indazole derivatives $7 \mathbf{a}-\mathbf{f}$, respectively (Scheme 1 ).

The reaction of any of compounds $3 \mathbf{a}-\mathbf{c}$ with two moles of hydroxylamine hydrochloride (8) in ethanol containing sodium acetate gave the 4,5,6,7-tetrahydrobenzo[c] isoxazol-3-yl)benzene derivatives 9a-c, respectively. The structures of compounds $\mathbf{9 a}-\mathbf{c}$ were established on the basis of analytical and spectral data. Thus, the ${ }^{1} \mathrm{H}$ NMR spectrum of $9 \mathbf{a}$ (as an example) showed the presence of the $\mathrm{OH}$ group at $\delta 9.52 \mathrm{ppm}\left(\mathrm{D}_{2} \mathrm{O}\right.$ exchangeable $)$ and two singlets at $\delta 2.47$ and $2.33 \mathrm{ppm}$ due to the presence of the two $\mathrm{CH}_{2}$ groups. On the other hand, the ${ }^{13} \mathrm{C}$ NMR spectrum gave signals at $\delta 140.1,137.6,127.8,126.5,124.0,121.2$ due to the phenyl and two isoxazole carbons.

The reaction of compound 1 with either 4-methyl-1-naphthalen-1-diazonium salt (10a) or 4-chloro-1 -naphthalen-1-diazonium salt (10b) gave the naphthylhydrazo derivatives 11a and $\mathbf{1 1} \mathbf{b}$, respectively. Compounds 11a,b reacted with elemental sulfur and either of malononitrile (4a) or ethyl cyanoacetate $(\mathbf{4 b})$ to give the naphthalen-1-yl)hydrazono)-6,7-dihydrobenzo[b]thiophene derivatives 12a-d, respectively. Compounds 11a,b were appropriate for Gewald's thiophene synthesis, thus the reaction of either of 11a or 11b with elemental sulfur and either of malononitrile (4a) or ethyl cyanoacetate $(\mathbf{4 b})$ gave

$$
\text { 3a-c }+\underset{8}{\mathrm{NH}_{2} \mathrm{OH} . \mathrm{HCl}} \stackrel{\mathrm{NaOAc}}{\stackrel{\mathrm{NtOH}}{\longrightarrow}}
$$<smiles>[X]c1ccc(-c2onc3c2C(=N)CC(C)(C)C3)cc1</smiles><smiles>CC1(C)CC(=O)CC(=O)C1</smiles>

1<smiles>[Y]c1ccc([N+]#[C-])c2ccccc12</smiles>

10a, $\mathrm{Y}=\mathrm{CH}_{3}$ b, $\mathrm{Y}=\mathrm{Cl}$

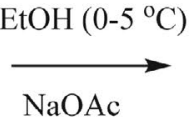<smiles>[Y]c1ccc(NN=C2C(=O)CC(C)(C)CC2=O)c2ccccc12</smiles>

11a, $\mathrm{Y}=\mathrm{CH}_{3}$ b, $\mathrm{Y}=\mathrm{Cl}$

$$
\begin{gathered}
11 \mathbf{a}, \mathbf{b}+\underset{\substack{! \\
\mathrm{R}}}{\mathrm{CH}_{2}}-\mathrm{CN}+\mathrm{S}_{8} \underset{\mathrm{Et}_{3} \mathrm{~N}}{\stackrel{\text { 1,4-dioxan }}{\longrightarrow}} \\
\text { 4a, } \mathrm{R}=\mathrm{CN} \\
\text { b, } \mathrm{R}=\mathrm{COOEt}
\end{gathered}
$$<smiles>[R]c1c(N)sc2c1C(=NNc1ccc([Y])c3ccccc13)C(=O)CC2(C)C</smiles>

12a, $\mathrm{Y}=\mathrm{CH}_{3}, \mathrm{R}=\mathrm{CN}$

b, $\mathrm{Y}=\mathrm{CH}_{3}, \mathrm{R}=\mathrm{COOEt}$

c, $\mathrm{Y}=\mathrm{Cl}, \mathrm{R}=\mathrm{CN}$

d, $\mathrm{Y}=\mathrm{Cl}, \mathrm{R}=\mathrm{COOEt}$

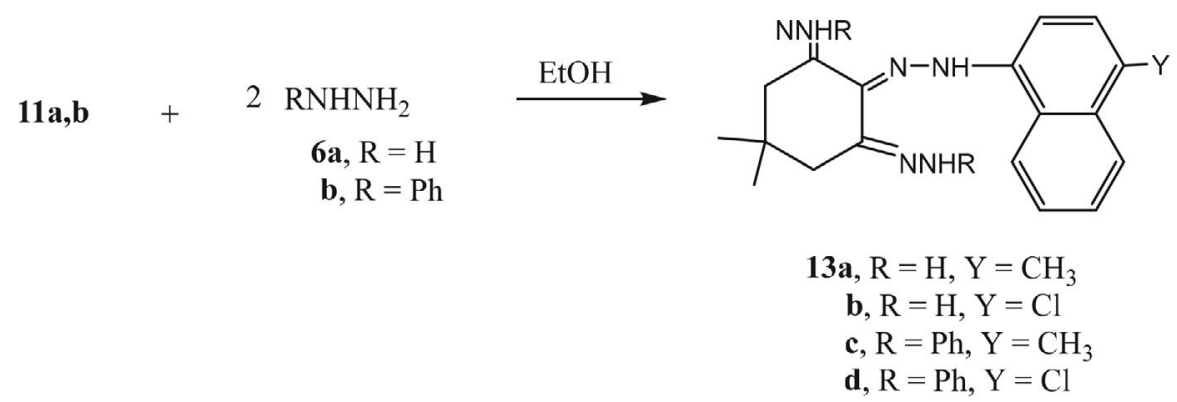

Scheme 2. Synthesis of compounds $9 a-c, 11 a, b, 12 a-d$ and 13a-d. 


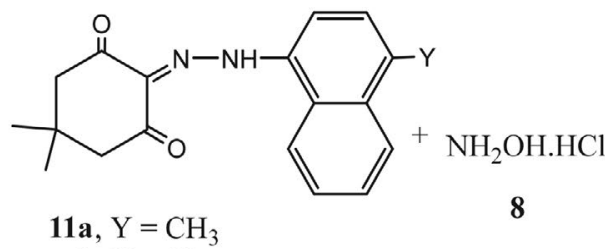<smiles>[Y]c1ccc(NN=C2C(=O)CC(C)(C(=O)OCC)CC2=NO)c2ccccc12</smiles>

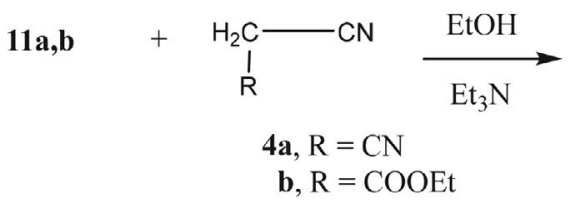<smiles>[R]C([Y19])=C1CC(C)(C)CC(=O)C1=NNc1ccc([Y])c2ccccc12</smiles><smiles></smiles>

Scheme 3. Synthesis of compounds 14a,b and 16a-d.

$$
\begin{gathered}
\text { 16a, } \mathrm{Y}=\mathrm{CH}_{3}, \mathrm{R}^{\prime}=\mathrm{NH} \\
\text { b, } \mathrm{Y}=\mathrm{CH}_{3}, \mathrm{R}^{\prime}=\mathrm{O} \\
\text { c, } \mathrm{Y}=\mathrm{Cl}, \mathrm{R}^{\prime}=\mathrm{NH} \\
\text { d, } \mathrm{Y}=\mathrm{Cl}, \mathrm{R}^{\prime}=\mathrm{O}
\end{gathered}
$$

the 6,7-dihydrobenzo[b]thiophene derivatives $\mathbf{1 3 a}-\mathbf{d}$, respectively (Scheme 2).

The reaction of either of $11 \mathbf{a}$ or $\mathbf{1 1 b}$ with hydroxylamine hydrochloride gave the cyclohexane-1,3-dione dioxime derivatives $\mathbf{1 4 a}$ and $\mathbf{1 4 b}$, respectively. On the other hand, the reaction of either of compound $11 \mathrm{a}$ or $11 \mathbf{b}$ with either of malononitrile (4a) or ethyl cyanoacetate (4b) gave the 2,3,5,6,7,8-hexahydrocinnoline derivatives $\mathbf{1 6 a}$ d, respectively (Scheme 3 ). The structures of the latter products were based on their respective analytical and spectral data. Thus, the ${ }^{1} \mathrm{H}$ NMR spectrum of $16 \mathbf{a}$ (as an example) showed the presence of a singlet at $\delta 8.25 \mathrm{ppm}$ due to the presence of the $\mathrm{NH}$ group, a multiplet at $\delta 7.53-$ $7.24 \mathrm{ppm}$ due to the presence of the naphthalene protons. In addition the ${ }^{13} \mathrm{C}$ NMR spectrum showed the presence of signals at $\delta 138.9,136.3,135.4,128.1,126.5,125.3,124.8$, $123.6,122.4,120.4$ due to the naphthalene carbons and two signals at $\delta 32.2,24.3$ for the two $\mathrm{CH}_{2}$ groups.

\section{Conclusion}

In conclusion, an efficient and practical synthesis of new series of heterocyclic compounds derived from dime-

done was carried out and the prepared compounds were characterized and their anti-proliferative activities were evaluated against the six cancer cell lines A549, HT-29, MKN-45, U87MG, SMMC-7721 and H460. The results showed that compounds $\mathbf{3 c}, \mathbf{5 b}, \mathbf{5 e}, \mathbf{5 f}, 7 \mathrm{c}, \mathbf{7 f}, \mathbf{9 c}, \mathbf{1 1 b}, \mathbf{1 2 c}$, 12d, 13b, 13d, 14b, 16c and 16d were the most potent compounds. On the other hand, compounds $\mathbf{5 b}, \mathbf{5 e}, \mathbf{5 f}, \mathbf{7 c}$, 11b, 12c, 12d, 13d, 14b, 16c and 16d were the most inhibitory active compounds against tyrosine kinases and compounds $5 \mathrm{~b}, 11 \mathrm{~b}, 12 \mathrm{~d}, 13 \mathrm{~d}, 14 \mathrm{~b}$ and $16 \mathrm{c}$ were the most potent against Pim-1 kinase.

\section{Acknowlegement}

R. M. Mohareb would like to thank the Alexander von Humboldt for affording him regular fellowships in Germany for doing research and completing this work.

\section{References}

1. L. G. Dutra, C. Saibert, D. S. Vicentini, M. M. Sa, J. Mol. Catal. A: Chemical 2014, 386, 35-41.

DOI:10.1016/j.molcata.2014.02.011 
2. J. S. Yadav, B. V. Subba, R. Rao, R. Narender, Tetrahedron Lett. 2009, 50, 3963-3965. DOI:10.1016/j.tetlet.2009.04.088

3. R. Kumar, K. Raghuvanshi, R. K. Verma, M. S. Singh, Tetrahedron Lett. 2010, 51, 5933-5936. DOI:10.1016/j.tetlet.2010.09.017

4. V. Nadaraj, S. T. Selvi, S. Mohan, Eur. J. Med. Chem., 2009, 44, 976-980. DOI:10.1016/j.ejmech.2008.07.004

5. M. L. Deb, P. J. Bhuyan, Tetrahedron Lett. 2005, 46, 6453-6456. DOI:10.1016/j.tetlet.2005.07.111

6. S. Chassaing, S. Specklin, J. M. Weibel, P. Pale, Tetrahedron 2012, 68, 7245-7273. DOI:10.1016/j.tet.2012.05.107

7. A. Amoozadeh, E. Tabrizian, S. Rahmani, Comptes Ren. Chim. 2015, 18, 848-857. DOI:10.1016/j.crci.2014.11.003

8. A. Ilangovan, S. Malayappasamy, S. Muralidharan, Chem. Cent. 2011, 5, 81. DOI:10.1186/1752-153X-5-81

9. A. M. Al-Majid, M. S. Islam, S. Barakat, N. J. Al-Qahtani, S. Yousef, M. I. Choudhary, Arab J. Chem. 2017, 10, 185-193. DOI:10.1016/j.arabjc.2014.04.008

10. J. M. Khurana, D. Magoo, Tetrahedron Lett. 2009, 52, 73007303. DOI:10.1016/j.tetlet.2009.10.032

11. H. Naeimi, Z. S. Nazif, J. Ind. Engin. Chem. 2014, 20, 10431049. DOI:10.1016/j.jiec.2013.06.041

12. K. Nikoofar, F. M. Yielzoleh, J. Saudi Chem. Soc. 2018, 22, 715-741. DOI:10.1016/j.jscs.2017.12.005

13. A. A. Abdelhamid, S. K. Mohamed, A. M. Maharramov, A. N. Khlalilov, M. A. Allahverdiev, J. Saudi Chem. Soc. 2014, 18, 474-478. DOI:10.1016/j.jscs.2011.10.005

14. M. L. Silva, R. R. Teixeira, L. A. Santos, F. T. Martins, T. C. Ramahho, J. Mol. Struct. 2018, 1156, 700-711. DOI:10.1016/j.molstruc.2017.11.105
15. C. Jiao, Y. J. Chao-guo, Chem. Res. Chin. Univ. 2011, 27, 49-53.

16. R. M. Mohareb, T. M. Klapötke, E. Reinhardt, Med. Chem. Research 2018, 27, 2494-2511.

DOI:10.1007/s00044-018-2252-3

17. R. M. Mohareb, F. Al-Omran, M. A. Abdelaziz, R. A. Ibrahim, Acta Chim. Slov. 2017, 64, 349-364. DOI:10.17344/acsi.2017.3200

18. R. M. Mohareb, P. A. Halim, Acta Chim. Slov. 2018, 65, 554568. DOI:10.17344/acsi.2017.4146

19. R. M. Mohareb, N. Y. Abdo, F. O. Al-Farouk, Acta Chim. Slov. 2017, 64, 117-128. DOI:10.17344/acsi.2016.2920

20. P. B. Jensen, T. Hunter, Nature 2001, 411, 355-365. DOI: $10.1038 / 35077225$

21. J. DiSalvo, M. L. Bayne, G. Conn, P. W. Kwok, P. G. Trivedi, D. D. Soderman, T. M. Palisi, K. A.Sullivan, K. A. Thomas, J. Biol. Chem. 1995, 270, 7717-7723. DOI:10.1074/jbc.270.13.7717

22. D. R. Senger, S. J. Galli, A. M. Dvorak, C. A. Perruzzi, V. S. Harvey, H. F. Dvorak, Science 1983, 219, 983-985. DOI:10.1126/science.6823562

23. N. Ferrara, Nat. Rev. Cancer, 2002, 2, 795-803. DOI: $10.1038 /$ nrc909

24. R. M. Mohareb, W. W. Wardakhan, R. A. Ibrahim, Med. Chem. Res. 2016, 25, 2187-2204.

DOI:10.1007/s00044-016-1654-3

25. A. M. Abdallah, R. M. Mohareb, E. M. Khalil, M. A. M Elshamy, Chem. Pharm. Bull. 2017, 65, 469-477. DOI:10.1248/cpb.c16-00925

26. W.Zhang, T. Ma, S. Li, Y. Yang, J. Guo, W. Yu, L. Kong, Eur. J. Med. Chem. 2017, 125, 538-550.

DOI:10.1016/j.ejmech.2016.09.068

\section{Povzetek}

Pri reakciji dimedona $\mathrm{z}$ arilaldehidi so nastali benzilidenski derivati 3a-c, ki smo jih v naslednji stopnji s heterociklizacijsko reakcijo pretvorili v pripojene tiofenske, pirazol izoksazolske in piridazinske derivate. Pripravljenim spojinam smo določili učinek na različne rakave celične linije ter tudi morebitno inhibicijo tirozin kinaze in Pim-1 kinaze. Za vse sintetizirane spojine smo določili inhibitorno aktivnost na A549 (nemikrocelični pljučni rak), H460 (človeški pljučni rak), HT-29 (rak človeškega debelega črevesa) in MKN-45 (rak človeškega želodca); foretinib smo uporabili kot pozitivno kontrolo pri MTT testiranju. Kot obetavne so se izkazale spojine 3c, 5b, 5e, 5f, 7c, 7f, 9c, 11b, 12c, 12d, 13b, 13d, 14b, 16c in 16d. Po drugi strani so se spojine 5b, 5e, 5f, 7c, 11b, 12c, 12d, 13d, 14b, 16c in 16d pokazale kot najbolj učinkoviti inhibitorji tirozin kinaze, spojine 5b, 11b, 12d, 13d, 14b in 16c pa so bile najbolj aktivne proti Pim-1 kinazi.

Except when otherwise noted, articles in this journal are published under the terms and conditions of the Creative Commons Attribution 4.0 International License 